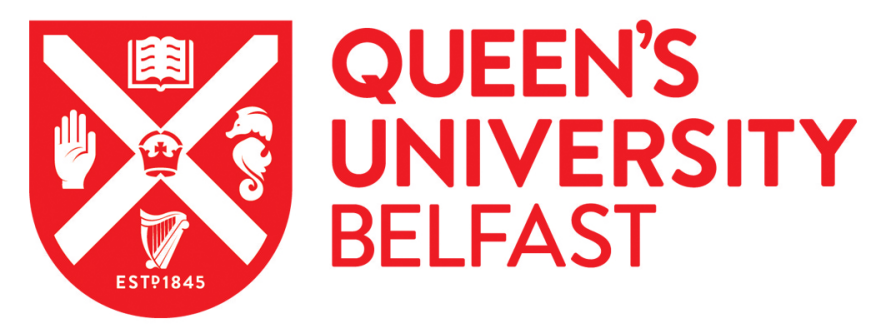

\title{
Market Economies with an Endogenous Social Division of Labour
}

Gilles, R. (2019). Market Economies with an Endogenous Social Division of Labour. International Economic Review, 60(2), 821-849. https://doi.org/10.1111/iere.12369

Published in:

International Economic Review

Document Version:

Peer reviewed version

Queen's University Belfast - Research Portal:

Link to publication record in Queen's University Belfast Research Portal

Publisher rights

( 2018 by the Economics Department of the University of Pennsylvania and the Osaka University Institute of Social and Economic Research Association. This work is made available online in accordance with the publisher's policies. Please refer to any applicable terms of use of the publisher.

\section{General rights}

Copyright for the publications made accessible via the Queen's University Belfast Research Portal is retained by the author(s) and / or other copyright owners and it is a condition of accessing these publications that users recognise and abide by the legal requirements associated with these rights.

Take down policy

The Research Portal is Queen's institutional repository that provides access to Queen's research output. Every effort has been made to ensure that content in the Research Portal does not infringe any person's rights, or applicable UK laws. If you discover content in the Research Portal that you believe breaches copyright or violates any law, please contact openaccess@qub.ac.uk. 


\title{
Market Economies with an Endogenous Social Division of Labour
}

\author{
Robert P. Gilles ${ }^{\dagger}$
}

September 2016

Revised: August 2017 and July 2018

\begin{abstract}
This paper considers a general equilibrium model of a competitive market economy in which production is conducted through an endogenous social division of labour. We represent economic decision makers as "consumer-producers", who consume as well as produce commodities. In this approach, the emergence of a non-trivial social division of labour is guided by Increasing Returns to Specialisation in production.

This paper investigates the properties of equilibria in economies satisfying Increasing Returns to Specialisation. We show that a perfectly competitive price mechanism induces a dichotomy of production and consumption at the level of the individual consumer-producer. In this context, under Increasing Returns to Specialisation, we show existence of competitive equilibria, the two fundamental theorems of welfare economics, and characterise these equilibria. Under certain conditions, markets are equilibrated through the adjustment of the social division of labour; therefore, prices are objectively determined by the production technologies on the supply side of the economy only.
\end{abstract}

Keywords: Social division of labour; Consumer-producer; Dichotomy of consumption and production; Competitive equilibrium.

JEL classification: D41, D51

*I am indebted to three referees, an associate editor and the editor of this journal for very detailed comments on previous drafts of this paper. I also thank Gabriella Graziano, Dimitrios Diamantaras, Achille Basile, Marialaura Pesce, Pieter Ruys and Owen Sims for their detailed feedback and discussion on previous drafts of this paper.

$\dagger$ Management School, The Queen’s University of Belfast, Riddel Hall, 185 Stranmillis Road, Belfast, BT9 5EE, UK. Email: r.gilles@qub.ac.uk 


\section{Specialisation and the social division of labour}

One of the oldest ideas explaining the human process of economic wealth creation is that this occurs through a functional social division of labour supported by a universal trade mechanism (Plato, 380 BCE; Aristotle, 350 BCE). As Smith (1776) already put forward, wealth creation in an economy with a social division of labour results from the interplay of two fundamental principles: (a) there are Increasing Returns to Specialisation and (b) full exploitation of these returns is possible through the principle of Mutual Gains From Trade. The emerging social division of labour not only acts as the main source of economic wealth creation, it also acts as an allocation mechanism. This paper investigates both aspects of the social division of labour in the context of a competitive market economy in which the law of one price is imposed on all production and trading processes.

The principle that human capital is more productive if fully specialised in the execution of a limited set of tasks or the production of a limited set of closely related commodities has not yet been explored fully in contemporary general equilibrium theory. This paper proposes formal descriptors of the property of Increasing Returns to Specialisation (IRSpec) that captures this fundamental idea. This conception compares to the notion of Increasing Returns to Scale (IRS), which has been the focus of research in general equilibrium approaches to economic wealth generation.

We show in this paper that the property of Increasing Returns to Specialisation supports the two fundamental functions of a social division of labour, namely the generation of economic wealth and the allocation of that generated wealth. Indeed, specialised economic agents interact through a competitive price mechanism that acts as a coordination device in the process of economic wealth creation as well as a mechanism to allocate the generated wealth through the social division of labour. To pursue this, we apply the approach developed in Yang (1988) and Yang and $\mathrm{Ng}$ (1993). ${ }^{1}$ This framework represents an economic decision maker as a "consumer-producer", who is endowed with consumptive as well as productive abilities. Yang presented this approach as an alternative to the formal (social) dichotomy of consumption and production that is at the centre of the Walrasian theory of a market economy.

However, the concept of Increasing Returns to Specialisation remains relatively unexplored in formal general equilibrium models based on the notion of a consumer-producer. Previously, Sun, Yang, and Zhou (2004) formalised a general equilibrium framework founded on the models of Yang $(1988,2001,2003)$ and Yang and $\mathrm{Ng}$ (1993). In this framework, production sets of individual consumer-producers are typically bounded as well as non-convex. These authors restrict their model to one based on home-based production only and investigate the existence of competitive equilibrium under well-specified transaction costs. This model omits the explicit formulation of either Increasing Returns to Scale or Increasing Returns to Specialisation and, as a result, does not investigate the consequences of these fundamental hypotheses. Also, the focus on home-based production excludes the trade of intermediary inputs as part of social production processes, an essential feature of general equilibrium models of production economies. In the present paper we try to address these omissions.

\footnotetext{
${ }^{1}$ Alternative explorations to model the idea of wealth creation through a social division of labour have been presented in, e.g., Young (1928); Stigler (1951); Blitch (1983); Kim (1989); Locay (1990) and Nakahashi and Feldman (2014). For a complete overview we also refer to Steinegger (2010) and Sun (2012).
} 
Our model and its main insights. We focus on the formalisation of the idea of Increasing Returns to Specialisation (IRSpec) at the level of the individual consumer-producer. A production set satisfies the IRSpec property if it is contained in the convex hull of production plans in which there is full specialisation in the production of a single commodity, subject to the free-disposal hypothesis in production. As mentioned above, Plato (380 BCE); Smith (1776); Yang (2001); Yao (2002) and Diamantaras and Gilles (2004) have hinted at this property, but it has not been formalised and developed further in the existing literature on mathematical models of market economies.

We extend the framework of Yang (1988); Yang and Ng (1993); Yang (2001) and Sun, Yang, and Zhou (2004) by assigning more general production sets, describing production plans founded on inputs and outputs, going beyond the limitations of home-based production only. This bridges this approach and the notion of a coalition-production economy (Hildenbrand, 1968, 1974). Furthermore, we abstract from transaction costs, thereby clarifying the pure effects of the properties of the consumer-producers' productive abilities we model. In the setting of this general model we offer a number of standard insights.

First, we establish that the competitive price mechanism induces a dichotomy between production and consumption decisions at the level of the individual consumer-producer (Theorem 2.6). Hence, the consumer-producer maximises her income through the selection of an optimal production plan and uses the generated income to acquire a preference-optimising consumption bundle.

Second, under IRSpec, individual consumer-producers specialise fully in the production of a single commodity as a consequence of the dichotomy between consumption and production decisions under a competitive price mechanism (Theorem 2.9). This simplifies significantly the insights of Yao (2002) and Diamantaras and Gilles (2004).

Third, in a continuum economy under standard assumptions on preferences and the boundedness of the production sets, we show that IRSpec implies the existence of a Walrasian equilibrium with strictly positive prices (Theorem 3.6). This existence result compares to the existence results established for coalition production economies (Hildenbrand, 1968, 1974; Sondermann, 1974; De Simone, 1997) and for economies with consumer-producers (Sun, Yang, and Zhou, 2004). ${ }^{2}$

Finally, we establish the two fundamental theorems of welfare economics for the formulated equilibrium concept. The first welfare theorem (Theorem 3.3) reflects a Smithian "invisible hand" that a competitive price guides consumer-producers to a socially optimal "role"-represented by a well-formulated production plan-in an optimally functioning social division of labour. We show that, under standard conditions, every Pareto optimal configuration consisting of an allocation and an assignment of production plans is supported as a quasi-equilibrium through a well-chosen lump-sum wealth transfer system (Theorem 3.4 (a)). Moreover, under IRSpec, every Pareto optimal configuration is supported as a full competitive equilibrium with strictly positive prices for a well-chosen lump-sum wealth transfer system (Theorem 3.4 (b)).

Further research should identify how these insights are modified if trade is subject to transaction costs. In particular, the interplay between Increasing Returns to Specialisation (IRSpec) and the presence of transaction costs should be investigated.

\footnotetext{
${ }^{2}$ We emphasise that our existence result is fundamentally different from the ones established by Suzuki (1995) and Toda (2002), who consider economies with non-convex consumption sets.
} 
The social division of labour as an allocation mechanism. The results established for a continuum economy with consumer-producers focus mainly on the endogenous emergence of a social division of labour. Therefore, these insights address the wealth creation processes in such an economy as much as the allocation of the generated wealth through that social division of labour. There is a further consequence to the IRSpec property in such an economy.

Indeed, IRSpec implies that besides equilibration through the price mechanism-as traditionally understood in Walrasian general equilibrium theory-there might arise equilibration through the endogenous adaptation of the social division of labour (Theorems 4.3 and 4.8). We show that this occurs in economies in which there are uniform production standards under full specialisationresulting into equivalent specialised abilities. In particular in these cases, the market prices are completely determined by the productive abilities of the consumer-producers in the economy and are as such independent of the demand side in the market system.

Therefore, under these conditions, free mobility and costless assumption of specialised roles in the social division of labour imply that competitive pressures equilibrate generated incomes as well as adjust the social division of labour to meet global market demand. This results in a fair allocation in which no agent envies any other (Corollary 4.4). Furthermore, equilibrium prices are fully determined by the production technologies in the economy-akin to a similar insight established for a Leontief economy with constant returns to scale.

Our notion of an economy with an endogenous social division of labour also encapsulates some aspects of production under Increasing Returns to Scale. However, our treatment of production is rather different from the models implementing Increasing Returns to Scale (Brown, 1991). Instead of considering the social organisation of production in "firms", our framework embeds production in a social division of labour that is fully atomised. Indeed, all economic agents are negligible consumer-producers and are endowed with flexible productive abilities. Natural limitations of human ability now imply that these individual production sets are possibly non-convex, but always bounded. This allows for a classical treatment of decision making with regard to production and the restoration of competitive equilibrium, even if individual production sets are non-convex.

Relationship to theories of economic growth. Recently, there has been a surging interest in the effects of increasing returns in wealth creation from new growth theory (Romer, 1986, 1987) to new international trade theory (Krugman, 1981, 1991, 2008; Gandolfo, 2014). None of these theories were developed within the context of a general equilibrium model of an economy with production subject to Increasing Returns to Specialisation (IRSpec). However, our work indicates that the properties debated in these theories can be implemented in the setting of a general equilibrium theory of an economy with consumer-producers.

New growth theory emerged from classical growth theory by taking account of innovation and Increasing Returns to Specialisation through modification of a standard Cobb-Douglas formulation of a production function. It has been shown by Yang and Borland (1991) and Yang and Ng (1993) that the general equilibrium framework founded on the modelling of agents as consumer-producers can be employed successfully to explain endogenous economic growth. An enhancement of the Yang-Borland model to incorporate IRSpec would make this link more explicit. 
New international trade theory has been founded on the incorporation of the modern approach to monopolistic competition developed by Dixit and Stiglitz (1977) to explain international trade by similarly developed countries. Our framework of an economy with multiple specialisation types (Definition 4.2) provides a general equilibrium framework to analyse international trade patterns between countries with different institutional governance structures. It clearly explains how similarly developed, advanced countries can establish non-trivial trade and the generation of significant wealth through the interplay of Increasing Returns to Specialisation (IRspec) and gains from (international) trade that is founded on perfect competition rather than monopolistic competition. We emphasise that this explanation is Smithian in nature rather than Ricardian (Buchanan and Yoon, 2002).

Outline of the paper. In Section 2 we set up the model of a consumer-producer and introduce formalisations of the notion of Increasing Returns to Specialisation (IRSpec). We investigate the dichotomy of consumption and production decisions at the level of the individual consumer-producer. We introduce two closely related formalisations of IRSpec. We subsequently show that, under IRSpec, production is normally fully specialised in a single output, confirming the insights of Yao (2002) and Diamantaras and Gilles (2004) without transaction costs.

In Section 3 we introduce a competitive equilibrium concept for which we establish the two fundamental theorems of welfare economics. Furthermore, we establish the existence of a competitive equilibrium under Increasing Returns to Specialisation.

Section 4 explores equilibration through the adjustment of the endogenous social division of labour. We show that this occurs if consumer-producers have equivalent productive standards under full specialisation.

All proofs of the main results are collected in the appendices.

\section{Consumer-producers}

Our approach is founded on the hypothesis that all agents participate directly in the production as well as the consumption of all economic commodities.

We consider $\ell \geqslant 2$ marketable commodities. The $\ell$-dimensional Euclidean space $\mathbb{R}^{\ell}$ now represents the commodity space and the consumption space is its nonnegative orthant $\mathbb{R}_{+}^{\ell}$. For $k=1, \ldots, \ell$ we denote by $e_{k}=(0, \ldots, 0,1,0, \ldots, 0)$ the $k$-th unit vector in $\mathbb{R}_{+}^{\ell}$ and by $e=(1, \ldots, 1)$ the bundle consisting of one unit of each commodity. Throughout, we use the notational convention for vector inequalities that $x \geqslant x^{\prime}$ if $x_{k} \geqslant x_{k}^{\prime}$ for all commodities $k=1, \ldots, \ell ; x>x^{\prime}$ if $x \geqslant x^{\prime}$ and $x \neq x^{\prime}$; and $x \gg x^{\prime}$ if $x_{k}>x_{k}^{\prime}$ for all commodities $k=1, \ldots, \ell$.

Every agent is a consumer-producer, endowed with consumptive as well as productive abilities, represented by a pair $(u, \mathcal{P})$, where $u: \mathbb{R}_{+}^{\ell} \rightarrow \mathbb{R}$ is a utility function representing the agent's consumptive preferences and $\mathcal{P} \subset \mathbb{R}^{\ell}$ is her production set consisting of production plans of outputs and inputs that the consumer-producer can execute. ${ }^{3}$

\footnotetext{
${ }^{3}$ Throughout, we use the notational convention that for two sets $S$ and $T, S \subset T$ stands for weak inclusion, i.e., $S$ is either a strict subset of $T$ or $S=T$.
} 


\subsection{Properties of production sets}

For every consumer-producer $(u, \mathcal{P})$, a production plan $y \in \mathcal{P}$ can be written as $y=y^{+}-y^{-}$where $y^{+}=y \vee 0 \in \mathbb{R}_{+}^{\ell}$ denotes the vector of outputs in this production plan and $y^{-}=(-y) \vee 0 \in \mathbb{R}_{+}^{\ell}$ denotes the vector of marketable inputs required for producing outputs $y^{+}$.

We distinguish two types of inputs: Marketable inputs, which are part of the $\ell$ commodities introduced above, and non-marketable inputs, which are assumed to be outside the realm of the commodity space. The approach to the modelling of production introduced here is, therefore, similar to the one detailed in Sondermann (1974, Section 2.1). ${ }^{4}$ We allow the possibility that all outputs are generated using non-marketable inputs only, i.e., $y^{+}>0$ and $y^{-}=0$. The latter case is the one explored mainly in the literature on economies with consumer-producers (Yang, 2001; Sun, Yang, and Zhou, 2004; Diamantaras and Gilles, 2004).

Definition 2.1 Consider a production set $\mathcal{P} \subset \mathbb{R}^{\ell}$. Then we introduce the following terminology:

(i) The production set $\mathcal{P}$ is regular if $\mathcal{P}$ is a closed set such that $0 \in \mathcal{P}$; it is comprehensive: ${ }^{5}$

$$
\mathcal{P}-\mathbb{R}_{+}^{\ell}=\left\{y-z \mid y \in \mathcal{P} \text { and } z \in \mathbb{R}_{+}^{\ell}\right\} \subset \mathcal{P}
$$

and it is bounded from above, i.e., there exists some $\bar{Q} \in \mathbb{R}^{\ell}$ such that $y \leqslant \bar{Q}$ for all $y \in \mathcal{P}$.

(ii) The production set $\mathcal{P}$ is delimited if there exists a non-empty compact subset $\overline{\mathcal{P}}$ of $\mathcal{P}$ such that $0 \in \overline{\mathcal{P}}$ and $\mathcal{P}=\overline{\mathcal{P}}-\mathbb{R}_{+}^{\ell}$.

(iii) The production set $\mathcal{P}$ is home-based if there exists a non-empty compact subset $\overline{\mathcal{P}}$ of $\mathcal{P}$ such that $\overline{\mathcal{P}} \subset \mathbb{R}_{+}^{\ell}$ and $\mathcal{P}=\overline{\mathcal{P}}-\mathbb{R}_{+}^{\ell}$.

A regular production set satisfies some properties accepted in general equilibrium models with production, namely the ability to cease production altogether and the assumption of free disposal in production. Note that regularity does not impose convexity, allowing production to exhibit non-convexities. Additionally, we require production to be bounded, which is a natural hypothesis due to the assumed size of a consumer-producer.

A delimited production set is based on a compact set of relevant production plans, subject to free disposal. Obviously, delimited production sets are regular. The difference between regular and delimited production sets is illustrated in Figure 1.

Home-based production is a special case of delimited production, reflecting the assumption that production is essentially based on the use of non-marketable inputs only. The relevant production plans now represent only output vectors that can be generated using non-marketable inputs and are collected in a compact set $\overline{\mathcal{P}} \subset \mathbb{R}_{+}^{\ell}$ with $0 \in \overline{\mathcal{P}}$ to which one applies free-disposal. We explicitly assume that under home production a consumer-producer can generate (marketable) commodities in positive quantities using non-marketable inputs only.

\footnotetext{
${ }^{4}$ Sondermann frames his discussion in the context of coalition production economies, i.e., productive abilities are assigned to coalitions of economic agents. For further discussions of coalition production economies we refer to Hildenbrand (1974); Sondermann (1974); Oddou (1982) and Basile (1993).

${ }^{5}$ For two sets $S$ and $T$, we let $S-T=\{x-y \mid x \in S$ and $y \in T\}$.
} 

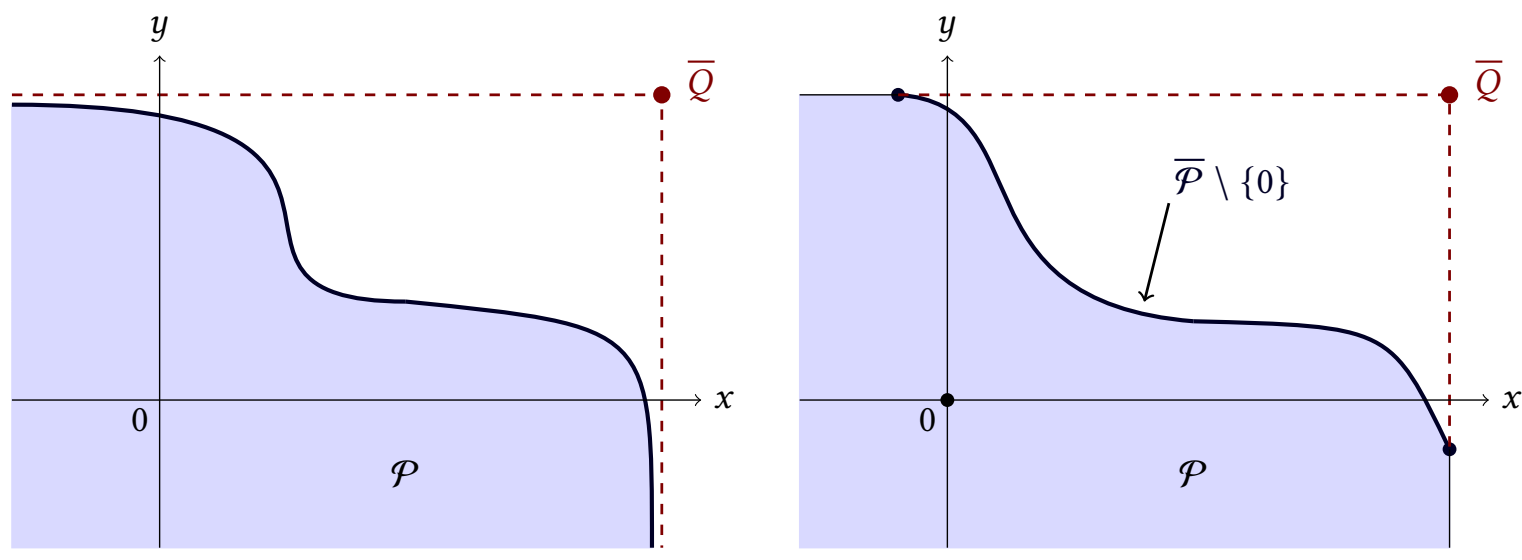

Figure 1: Comparing regular and delimited production sets.

Figure 2 illustrates home-based production. In the left panel the production set is founded on exactly 4 home production plans, using non-marketable inputs to produce positive quantities of two marketable commodities $X$ and $Y$. In the right panel the home-based production set is based on a compact curve in $\mathbb{R}_{+}^{2}$ denoted by $\overline{\mathcal{P}}$. In both cases the production set is constructed by applying the free-disposal property to all home production plans represented in $\overline{\mathcal{P}}$.
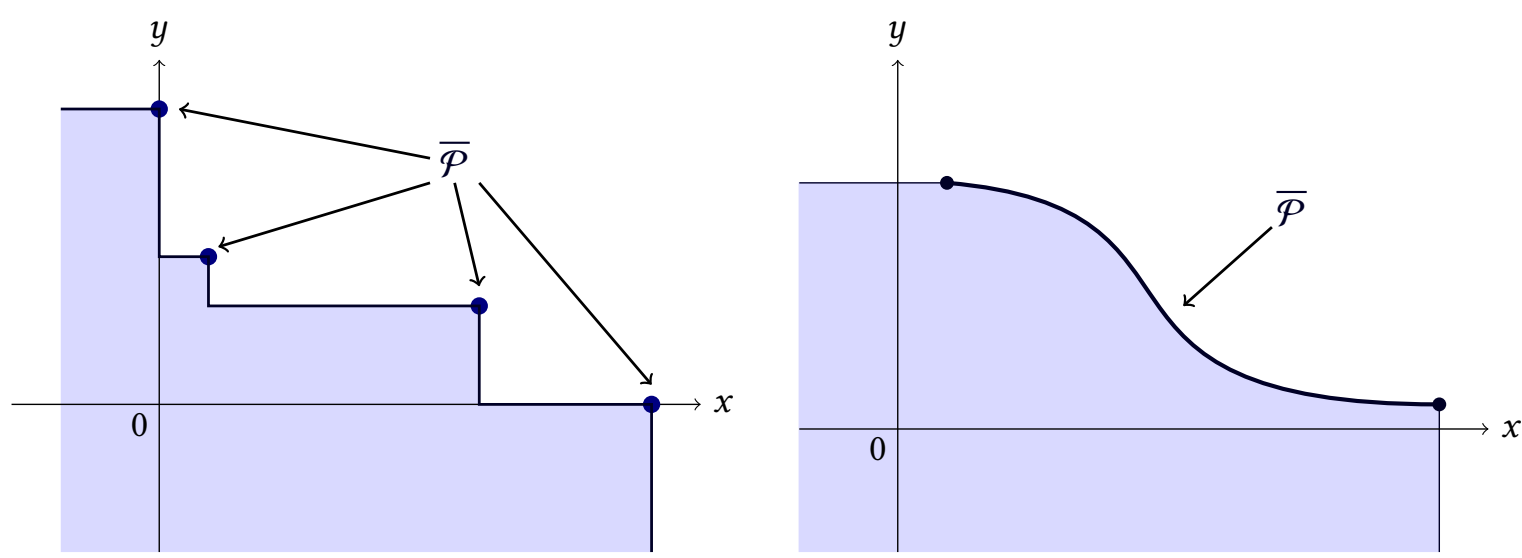

Figure 2: Illustrations of home-based production sets.

Some regularity assumptions. Throughout this paper we impose standard regularity conditions on the preferences as well as the productive abilities in this economy.

Axiom 2.2 For every consumer-producer represented by the pair $(u, \mathcal{P})$ we assume the following properties:

(i) The utility function $u: \mathbb{R}_{+}^{\ell} \rightarrow \mathbb{R}$ is continuous on the consumption space $\mathbb{R}_{+}^{\ell}$ and

(ii) The production set $\mathcal{P} \subset \mathbb{R}^{\ell}$ is regular.

Additional assumptions are used in various theorems that we derive throughout this paper. In particular, we may impose that preferences satisfy certain standard monotonicity properties: A 
utility function $u: \mathbb{R}_{+}^{\ell} \rightarrow \mathbb{R}$ satisfies local non-satiation if for every bundle $x_{1} \in \mathbb{R}_{+}^{\ell}$ and every $\varepsilon>0$ there exists some bundle $x_{2} \in \mathbb{R}_{+}^{\ell}$ such that $\left\|x_{1}-x_{2}\right\|<\varepsilon$ and $u\left(x_{2}\right)>u\left(x_{1}\right)$; a utility function is non-decreasing if $u\left(x_{2}\right) \geqslant u\left(x_{1}\right)$ for all $x_{2} \geqslant x_{1}$; a utility function $u$ is monotone if $u\left(x_{2}\right)>u\left(x_{1}\right)$ for all $x_{2} \gg x_{1}$; and a utility function $u$ is strictly monotone if $u\left(x_{2}\right)>u\left(x_{1}\right)$ for all $x_{2}>x_{1}$.

Production functions. Yang (2001); Yao (2002); Cheng and Yang (2004) and Diamantaras and Gilles (2004) assume that production sets are generated by production functions. The formulation of a consumer-producer as a pair $(u, \mathcal{P})$ satisfying Axiom 2.2 also captures the case that production is based on the allocation of one unit of non-marketable labour time over $\ell$ different (home) production processes, each generating the output of a marketable commodity. ${ }^{6}$

Example 2.3 For every commodity $k \in\{1, \ldots, \ell\}$, let $\varphi_{k}:[0,1] \rightarrow \mathbb{R}_{+}$be a production function converting a certain fraction of one unit of invested labour time in the output of the $k$-th commodity. We impose that $\varphi_{k}$ is continuous and non-decreasing, that $\varphi_{k}\left(L_{k}\right)>0$ for all $L_{k}>0$, and that $\varphi_{k}(0)=0$. Now let $\phi(L)=\left(\varphi_{1}\left(L_{1}\right), \ldots, \varphi_{\ell}\left(L_{\ell}\right)\right)$ for any labour time vector $L \in[0,1]^{\ell}$ such that $\sum_{k} L_{k}=1$. Now define

$$
\mathcal{P}(\phi)=\overline{\mathcal{P}}(\phi)-\mathbb{R}_{+}^{\ell} \subset \mathbb{R}^{\ell}
$$

where

$$
\overline{\mathcal{P}}(\phi)=\left\{\phi\left(L_{1}, \ldots, L_{\ell}\right) \mid \sum_{k=1}^{\ell} L_{k}=1\right\} \subset \mathbb{R}_{+}^{\ell} .
$$

Now, $\mathcal{P}(\phi)$ is a home-based production set. Indeed, $0 \in \mathcal{P}(\phi)$ and $\mathcal{P}(\phi)$ is bounded from above by $\left(\varphi_{1}(1), \ldots, \varphi_{\ell}(1)\right) \gg 0$ as well as comprehensive. Also, due to the scalability of labour time, $\phi\left(\frac{1}{\ell}, \ldots, \frac{1}{\ell}\right) \in \mathcal{P}(\phi) \cap \mathbb{R}_{++}^{\ell}$, which implies that the agent can produce all marketable commodities in positive quantities.

\subsection{The consumer-producer's problem}

A consumer-producer represented by $(u, \mathcal{P})$ participates in a system of perfectly competitive markets and faces a price vector $p \in \mathbb{R}_{+}^{\ell}$. Consequently, she optimises her utility over the set of feasible consumption-production plans with the goal to maximise consumptive satisfaction. This optimisation problem is denoted as the "consumer-producer problem":

Axiom 2.4 For any $p>0$, a consumer-producer $(u, \mathcal{P})$ aims to select a consumption plan $\hat{x} \in \mathbb{R}_{+}^{\ell}$ and production plan $\hat{y} \in \mathcal{P}$ such that $(\hat{x}, \hat{y})$ solves

$$
\max \left\{u(x) \mid x \in \mathbb{R}_{+}^{\ell}, y \in \mathcal{P} \text { and } p \cdot x \leqslant p \cdot y\right\} .
$$

\footnotetext{
${ }^{6} \mathrm{We}$ emphasise that the referred framework is based on the investment of labour time in the production of the various commodities. The main difference between the construction developed in Example 2.3 below and the model referred to from the literature is that we assume that labour time cannot be converted to leisure time and leisure time is not featured explicitly as an input in the utility function.
} 
In this statement, $\hat{x} \in \mathbb{R}_{+}^{\ell}$ is agent $a$ 's optimal consumption bundle, while $\hat{y}$ is an optimal production plan to support that consumption bundle. Now, $t=\hat{y}-\hat{x} \in \mathbb{R}^{\ell}$ is the vector of net trades submitted to the market. The next lemma is straightforward and, therefore, a proof is omitted.

Lemma 2.5 If $(u, \mathcal{P})$ satisfies Axiom 2.2, the consumer-producer problem (4) admits a solution for every strictly positive price vector $p \gg 0$.

The dichotomy of consumption and production. The consumer-producer problem can be transformed into a two-stage maximisation problem that separates the production decision from the consumption decision. In the first stage, a production plan is selected that maximises the income generated for the given market prices. In the second stage, this maximal income is used to acquire a utility maximising final consumption bundle. This establishes a dichotomy of the production and consumption decisions under a price mechanism at the level of the individual economic agent. ${ }^{7}$

\section{Theorem 2.6 - Dichotomy of consumption and production}

Let $(u, \mathcal{P})$ represent a consumer-producer. Under the regularity properties stated in Axiom 2.2 and the additional assumption that $u$ is monotone, for every positive price vector $p>0$, the consumer-producer problem (4) is equivalent to the following two-stage problem:

Income maximisation: The consumer-producer first solves

$$
\max \{p \cdot y \mid y \in \mathcal{P}\}
$$

Denote the solution of (5) by

$$
\Omega(p)=\arg \max \{p \cdot y \mid y \in \mathcal{P}\} \subset \mathcal{P}
$$

and

$$
I(p)=\max \{p \cdot y \mid y \in \mathcal{P}\}=p \cdot \Omega(p) .
$$

Standard demand problem: The consumer-producer subsequently solves a standard demand problem formulated as

$$
\max \{u(x) \mid p \cdot x \leqslant I(p)\}
$$

For a proof of this assertion we refer to Appendix A.

Wen (1998b, Proposition 1) shows that the dichotomy of consumption and production cannot be extended to situations with transaction costs as set out in Yang (2001).

\footnotetext{
${ }^{7}$ We emphasise that this "weak" dichotomy is different from a "social" dichotomy that is imposed through the social organisation of production in general equilibrium models of Walrasian economies with production.
} 


\subsection{Increasing Returns to Specialisation}

Our aim is to formally express the principle that production is subject to Increasing Returns to Specialisation, representing the human ability to learn to produce outputs more effectively when specialising in certain tasks (Smith, 1776, Chapter 1). In our framework such specialisation is captured by the notion of a "full specialisation" production plan, in which an agent produces only one output and uses any other commodity as an input only. Our formalisation of Increasing Returns to Specialisation states that a complete set of full specialisation production plans form the corner points of the production set available to the consumer-producer.

Definition 2.7 Consider a regular production $\operatorname{set} \mathcal{P} \subset \mathbb{R}^{\ell}$.

Full specialisation: A production plan $z^{k} \in \mathcal{P}$ is a full specialisation production plan for commodity $k \in\{1, \ldots, \ell\}$ if there exists some positive output quantity $Q^{k}>0$ and some input vector $y^{k} \in \mathbb{R}_{+}^{\ell}$ such that $y_{k}^{k}=0, z^{k+}=Q^{k} e_{k}$ and $z^{k-}=y^{k}$.

WIRSpec: The production set $\mathcal{P}$ exhibits Weakly Increasing Returns to Specialisation iffor every commodity $k \in\{1, \ldots, \ell\}$ there exists some full specialisation production plan $z^{k} \in \mathcal{P}$ such that

$$
\mathcal{P} \subset \operatorname{Conv}\left\{z^{1}, \ldots, z^{\ell}\right\}-\mathbb{R}_{+}^{\ell}
$$

where Conv $S=\{\lambda x+(1-\lambda) y \mid x, y \in S$ and $0 \leqslant \lambda \leqslant 1\}$ denotes the convex hull of a set $S \subset \mathbb{R}^{\ell}$.

SIRSpec: The regular production set $\mathcal{P}$ exhibits Strongly Increasing Returns to Specialisation if $\mathcal{P}$ exhibits WIRSpec for a corresponding set of full specialisation production plans $Q=$ $\left\{z^{1}, \ldots, z^{\ell}\right\}$ introduced above such that $\mathcal{P} \cap(\operatorname{Conv} Q)=Q$.

The definition above formalises the idea that specialising in the production of a single commodity results in the maximal output of that particular commodity. In this formalisation we assume that the consumer-producer is able to fully specialise in any of the $\ell$ marketable commodities under consideration. This is a strong assumption, requiring that the consumer-producer has specialised productive abilities in all marketable commodities.

From Definition 2.7, WIRSpec implies that all full specialisation production plans in $Q=$ $\left\{z^{1}, \ldots, z^{\ell}\right\}$ are necessarily on the boundary of the production set $\mathcal{P}: z^{k}=Q^{k} e_{k}-y^{k} \in \partial \mathcal{P}$ for every $k \in\{1, \ldots, \ell\}$. As such, the property that $Q \subset \mathcal{P} \subset \operatorname{Conv} Q-\mathbb{R}_{+}^{\ell}$ implies that the full specialisation production plans in $Q$ are exactly those production plans in which all inputs are used optimally. These are natural candidates for solutions to the income maximisation problem.

Illustration of WIRSpec. A production set satisfying WIRSpec is illustrated in Figure 3. It considers a delimited production set $\mathcal{P}$ based on a finite set of 5 production plans $\overline{\mathcal{P}}$ as depicted. The two most extreme production plans $z^{1}$ and $z^{2}$ in $\overline{\mathcal{P}}$ form a set $Q=\left\{z^{1}, z^{2}\right\}$ and span $\operatorname{Conv} Q$. As such, in the two production plans $z^{1}$ and $z^{2}$ the production of a single output is most efficient: In $z^{2}$, the output of commodity $X$ is maximised, while in $z^{1}$ the output of commodity $Y$ is maximised. 


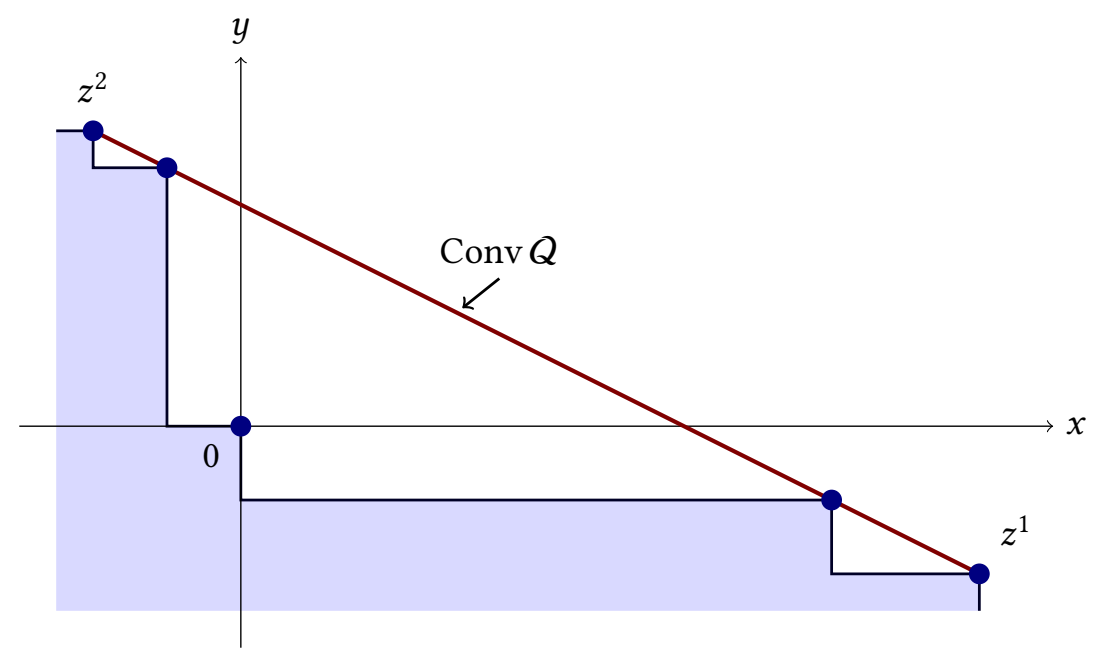

Figure 3: Illustration of a production set satisfying WIRSpec.

Illustration of SIRSpec. SIRSpec imposes that there is a strict productivity increase from specialising in the production of a single output in the sense that maximal income levels can only be attained through full specialisation in the production of that particular commodity. This is illustrated in Figure 4, which is a modification of the illustration in Figure 3.

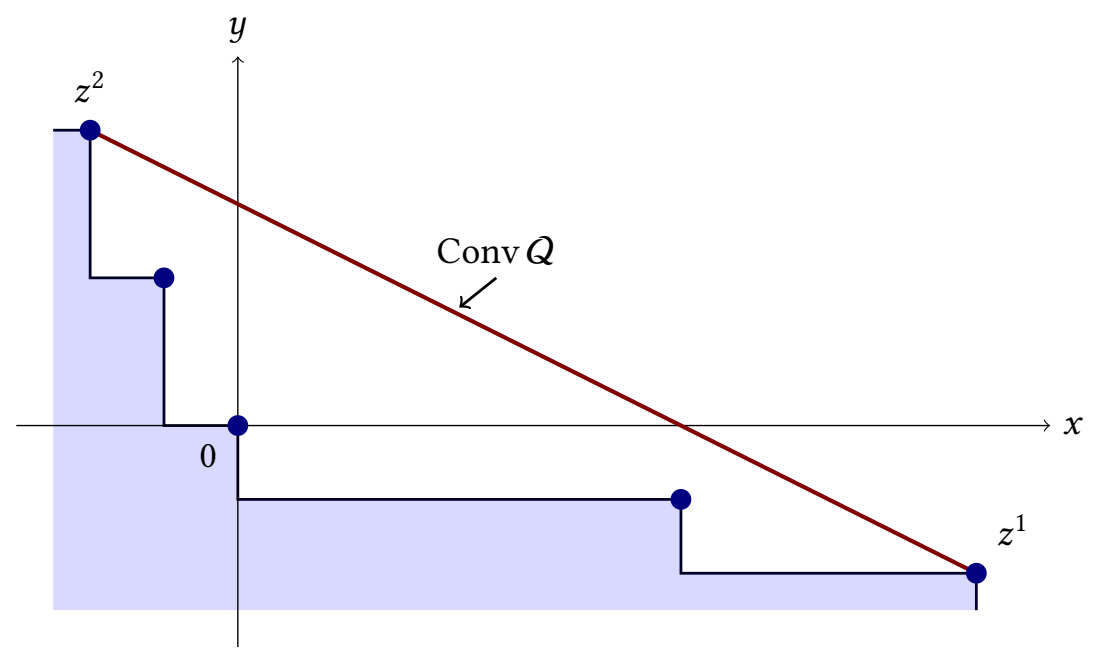

Figure 4: Illustration of a production set satisfying SIRSpec.

Some properties of IRSpec production sets. The next proposition collects some properties of production sets satisfying WIRSpec and SIRSpec. In particular, SIRSpec implies that strictly positive collective output levels of all commodities can be achieved by mixing well-chosen full specialisation production plans. A proof is presented in Appendix B.

\section{Proposition 2.8}

(a) If a production set $\mathcal{P}$ satisfies WIRSpec, then it is delimited. 
(b) If a production set $\mathcal{P}$ is home-based, then $\mathcal{P}$ satisfies WIRSpec if and only if there exist output quantities $Q^{k}>0$ for all $k \in\{1, \ldots, \ell\}$ with $Q \subset \mathcal{P} \subset \operatorname{Conv} Q-\mathbb{R}_{+}^{\ell}$ where $Q=\left\{Q^{1} e_{1}, \ldots, Q^{\ell} e_{\ell}\right\}$.

(c) If a production set $\mathcal{P} \subset \mathbb{R}^{\ell}$ satisfies SIRSpec, then $\operatorname{Conv} \mathcal{P} \cap \mathbb{R}_{++}^{\ell} \neq \varnothing$.

Next we extend the dichotomy of production and consumption (Theorem 2.6). We show that IRSpec implies that-as expected-the consumer-producer's income is maximised in a full-specialisation production plan. This theorem compares to similar specialisation results stated in Wen (1998a,b), Yao (2002, Theorem 2) and Diamantaras and Gilles (2004, Theorem 5). ${ }^{8}$

\section{Theorem 2.9 - Specialisation Theorem}

(a) IfP satisfies WIRSpec, then for any solution of the consumer-producer problem (4) for $p>0$, there exists a solution in which the consumer-producer produces no more than one commodity and obtains at least as much utility.

(b) IfP satisfies SIRSpec, then every solution of the consumer-producer problem (4) for $p>0$ is such that the consumer-producer produces no more than one commodity.

A proof of Theorem 2.9 is provided in Appendix $C$ of this paper.

The notion of Increasing Returns to Specialisation (IRSpec) as formulated here generalises the well-known notion of convexity of production functions (Example 2.3), which in turn implies that the generated production set satisfies Increasing Returns to Scale (IRS). For a proof of the following assertions we refer to Appendix D.

Proposition 2.10 Consider the model of production developed in Example 2.3.

(a) If all production functions $\varphi_{k}(k=1, \ldots, \ell)$ are convex, then $\mathcal{P}(\phi)$ satisfies WIRSpec.

(b) If all production functions $\varphi_{k}(k=1, \ldots, \ell)$ are convex and for at least one $k^{\prime}$ the production function $\varphi_{k^{\prime}}$ is strictly convex, then $\mathcal{P}(\phi)$ satisfies SIRSpec.

\section{Market economies and competitive equilibria}

We consider a model of an economy in which all agents are consumer-producers and simultaneously make decisions regarding consumption and production under a given vector of competitive market prices. Our definition subsumes the continuity and regularity properties stated in Axiom 2.2.

Definition 3.1 An economy with $\ell$ commodities is a triple $\mathbb{E}=\langle(A, \Sigma, \mu), u, \mathcal{P}\rangle$ where

(i) $(A, \Sigma, \mu)$ is a complete probability space of consumer-producers such that every a $\in A$ is represented by a pair $\left(u_{a}, \mathcal{P}_{a}\right)$;

\footnotetext{
${ }^{8}$ The main difference between the framework developed in the current paper and the referred contributions is that these papers consider as well the effects of transactions costs on the production and consumption decisions of consumerproducers. The introduction of transaction costs reduces the clarity of the analysis of specialisation decisions in the consumer-producer problem.
} 
(ii) The function $u: A \times \mathbb{R}_{+}^{\ell} \rightarrow \mathbb{R}$ assigns a continuous utility function $u_{a}$ to every $a \in A$ such that for every consumption bundle $x \in \mathbb{R}_{+}^{\ell}$ the function $u(\cdot, x): A \rightarrow \mathbb{R}$ is measurable on $(A, \Sigma)$;

(iii) The correspondence $\mathcal{P}: A \rightarrow 2^{\mathbb{R}^{\ell}}$ assigns a regular production set $\mathcal{P}_{a} \subset \mathbb{R}^{\ell}$ to every agent $a \in A$ and has a measurable graph in $\Sigma \otimes \mathcal{B}\left(\mathbb{R}^{\ell}\right)$, and;

(iv) There exists an integrable upper bound $\bar{Q}: A \rightarrow \mathbb{R}^{\ell}$ such that for almost every $a \in A: y \leqslant \bar{Q}(a)$ for every production plan $y \in \mathcal{P}_{a}$.

An economy $\mathbb{E}=\langle(A, \Sigma, \mu), u, \mathcal{P}\rangle$ is a continuum economy if the complete probability space of consumer-producers $(A, \Sigma, \mu)$ is atomless.

We emphasise that this definition of an economy imposes that preferences are assumed to be continuous and production sets to be regular. Furthermore, we incorporate standard measurability properties of preferences and the production correspondence. Finally, productive abilities are bounded from above by an integrable upper bound.

An allocation in the economy $\mathbb{E}=\langle(A, \Sigma, \mu), u, \mathcal{P}\rangle$ is a pair $(f, g)$ where $f: A \rightarrow \mathbb{R}_{+}^{\ell}$ is an integrable assignment of consumption bundles and $g: A \rightarrow \mathbb{R}^{\ell}$ is an integrable selection of the production correspondence $\mathcal{P}$. Thus, an allocation $(f, g)$ consists of an assignment of consumption bundles $f$ and an assignment of production plans $g$.

An allocation $(f, g)$ is feasible if all allocated consumption bundles are covered exactly by the produced quantities of all commodities, i.e.,

$$
\int f d \mu=\int g d \mu
$$

An equilibrium can now be introduced as a feasible allocation that is supported by a price vector for which almost all consumer-producers select optimal consumption and production plans.

Definition 3.2 Consider an economy $\mathbb{E}=\langle(A, \Sigma, \mu), u, \mathcal{P}\rangle$.

An allocation $(f, g)$ is a competitive equilibrium in $\mathbb{E}$ if $(f, g)$ is feasible and there exists some positive price vector $p>0$ such that for almost every agent $a \in A,(f(a), g(a))$ solve $a$ 's consumer-producer problem (4) for $p$ (Axiom 2.4).

\subsection{Pareto optimality and the Welfare Theorems}

Traditionally, the so-called "Fundamental Theorems of Welfare Economics" investigate the relationship between social or Pareto optimality and market equilibrium.

Formally, an allocation $(f, g)$ in an economy $\mathbb{E}$ is Pareto optimal if $(f, g)$ is feasible and there is no alternative feasible allocation $\left(f^{\prime}, g^{\prime}\right)$ such that $u_{a}\left(f^{\prime}(a)\right) \geqslant u_{a}(f(a))$ for almost all agents $a \in A$ and there exists some non-negligible coalition $S \in \Sigma$ with $\mu(S)>0$ such that $u_{a}\left(f^{\prime}(a)\right)>u_{a}(f(a))$ for all $a \in S$. We show that every competitive equilibrium is Pareto optimal in our framework.

\section{Theorem 3.3 - First Welfare Theorem}

Let $\mathbb{E}=\langle(A, \Sigma, \mu), u, \mathcal{P}\rangle$ be an economy. If almost every agent $a \in A$ has a monotone utility function $u_{a}$, then every competitive equilibrium in $\mathbb{E}$ is Pareto optimal. 
A proof of this theorem is relegated to Appendix E.

The Second Welfare Theorem asserts that every Pareto optimal allocation can be supported as an equilibrium through an appropriate re-allocation of initial resources. In the extension of the Second Welfare Theorem to our setting, we use the notion of a tax-subsidy scheme or lump-sum wealth transfer system $\tau: A \rightarrow \mathbb{R}$ to re-allocate wealth among agents. Here, for $a \in A, \tau(a)>0$ represents a tax, while $\tau(a)<0$ denotes a subsidy.

\section{Theorem 3.4 - Second Welfare Theorem}

Let $\mathbb{E}=\langle(A, \Sigma, \mu), u, \mathcal{P}\rangle$ be a continuum economy and let $(f, g)$ be a Pareto optimal allocation in $\mathbb{E}$.

(a) If almost every agent $a \in A$ has a utility function $u_{a}$ that satisfies local non-satiation, then there exists an integrable transfer system $\tau: A \rightarrow \mathbb{R}$ with $\int \tau d \mu=0$ and a price vector $p>0$ such that for almost every $a \in A$ :

$$
\tau(a) \leqslant \sup p \cdot \mathcal{P}_{a} \quad \text { and } p \cdot f(a)+\tau(a)=p \cdot g(a)=\sup p \cdot \mathcal{P}_{a}
$$

as well as

$$
u_{a}(x) \geqslant u_{a}(f(a)) \text { implies that } p \cdot x+\tau(a) \geqslant \sup p \cdot \mathcal{P}_{a} .
$$

(b) If almost every agent $a \in A$ has a strictly monotone utility function $u_{a}$ and a production set $\mathcal{P}_{a}$ satisfying SIRSpec, then there exists an integrable transfer system $\tau: A \rightarrow \mathbb{R}$ with $\int \tau d \mu=0$ and a strictly positive price vector $p \gg 0$ such that for almost every $a \in A$ :

$$
\tau(a) \leqslant \max p \cdot \mathcal{P}_{a} \quad \text { and } p \cdot f(a)+\tau(a)=p \cdot g(a)=\max p \cdot \mathcal{P}_{a}
$$

as well as

$$
u_{a}(x)>u_{a}(f(a)) \text { implies that } p \cdot x+\tau(a)>p \cdot g(a) \text {. }
$$

For a proof of Theorem 3.4 we refer to Appendix F.

\subsection{Existence of competitive equilibria}

The existence of competitive equilibria in our framework compares to the existence of equilibria in coalition-production economies. Hildenbrand (1974, Chapter 4) established the existence of quasiequilibria, which was subsequently strengthened to the existence of full equilibria by Suzuki (1995), De Simone (1997) and Toda (2002) under various hypotheses. We show that in our setting competitive equilibria can quite straightforwardly be shown to exist if the economy satisfies Strongly Increasing Returns to Specialisation (SIRSpec), avoiding the additional restricting hypotheses introduced by these authors. As expected, the introduction of Increasing Returns to Specialisation makes existence difficult to establish in countably infinite and finite economies. 


\section{Example 3.5 - Non-existence of competitive equilibria in a countable economy. ${ }^{9}$}

We consider an economy with two commodities $X$ and $Y$ and a countable number of economic agents, who are identical in consumptive and productive capabilities, but who are heterogenous through their relative size.

In particular, consider an agent population $A=\mathbb{N}$ with $\Sigma=2^{\mathbb{N}}$ and $\mu(S)=\sum_{a \in S} \lambda_{a}$ where $\lambda_{1}=\frac{2}{3}$ and $\lambda_{a}=\frac{1}{3 \cdot 2^{a-1}}$ for all $a \in \mathbb{N} \backslash\{1\}$. Obviously, $\mu(A)=1$ since $\lambda$ is a convergent series.

Furthermore, for every $a \in A: \mathscr{P}_{a}=\{(1,0),(0,1)\}$ and $u_{a}(x, y)=x y$.

Claim: This economy does not admit a competitive equilibrium.

Indeed, since both commodities are necessities for all economic agents, both need to be produced in positive quantities. From the income maximisation problem stated in Theorem 2.6, this is only possible if $p_{x}=p_{y}=p>0$ and the generated income is $I_{a}=p$ for all $a \in A$. This implies for all $a \in A$ the optimal consumption bundle is given by $f(a)=\left(\frac{1}{2}, \frac{1}{2}\right)$. Thus, market demand is $\int f d \mu=\left(\frac{1}{2}, \frac{1}{2}\right)$, which cannot be matched by market supply due to the fact that the production choice of agent 1 would generate an excess supply of that particular commodity. Hence, there is no equilibrium in this economy.

Under Strongly Increasing Returns to Specialisation (SIRSpec), the existence of a non-trivial competitive equilibrium can be established in a continuum economy. A proof of this theorem is provided in Appendix G of this paper.

\section{Theorem 3.6 - Existence Theorem}

Suppose that $\mathbb{E}=\langle(A, \Sigma, \mu), u, \mathcal{P}\rangle$ is a continuum economy such that almost every agent a $\in A$ has a strictly monotone utility function $u_{a}$ and a production set $\mathcal{P}_{a}$ that satisfies SIRSpec. Then there exists a competitive equilibrium in $\mathbb{E}$ with $f(a)>0$ for almost all agents $a \in A$.

We remark that the assumptions on the production sets in the assertion of Theorem 3.6 combine delimited production sets with an integrable upper bound, introduced as Definition 3.1(iv). This delimited integrable boundedness condition is weaker than the uniform boundedness condition (2d) in De Simone (1997, page 254) as well as the boundedness hypothesis imposed as Assumption 2 in Sun, Yang, and Zhou (2004, page 241) to guarantee existence of competitive equilibria in similarly formulated economies with agent-based production. Therefore, our existence theorem does not follow directly from the results stated in these contributions. ${ }^{10}$

The assertion of Theorem 3.6 that there exists an equilibrium with non-trivial consumption no longer holds if we weaken the requirement on the production sets to WIRSpec instead of SIRSpec. Indeed, the next example shows that under WIRSpec competitive equilibria might exist in which no meaningful consumption is achieved, even though all consumer-producers are engaged in productive activities and there is trade of all commodities in the economy.

\footnotetext{
${ }^{9}$ I thank a referee of this journal for pointing out this simple counterexample.

${ }^{10}$ Furthermore, we note that Sun, Yang, and Zhou (2004) impose a different structure of the productive abilities of the consumer-producers, which is founded on home-based production through the single input of labour only similar to the structure introduced in Example 2.3.
} 
Example 3.7 Consider an economy with two commodities $X$ and $Y$ and a continuum $(A, \Sigma, \mu)$ of consumer-producers. Let all consumer-producers have identical production sets given by $\mathcal{P}=$ $\{(1,-1),(0,0),(-1,1)\}-\mathbb{R}_{+}^{2}$. This production set reflects that each consumer-producer can convert one commodity into the other, or be productively inactive. Clearly, the production set $\mathcal{P}$ satisfies WIRSpec, but not SIRSpec, as illustrated in Figure 5.

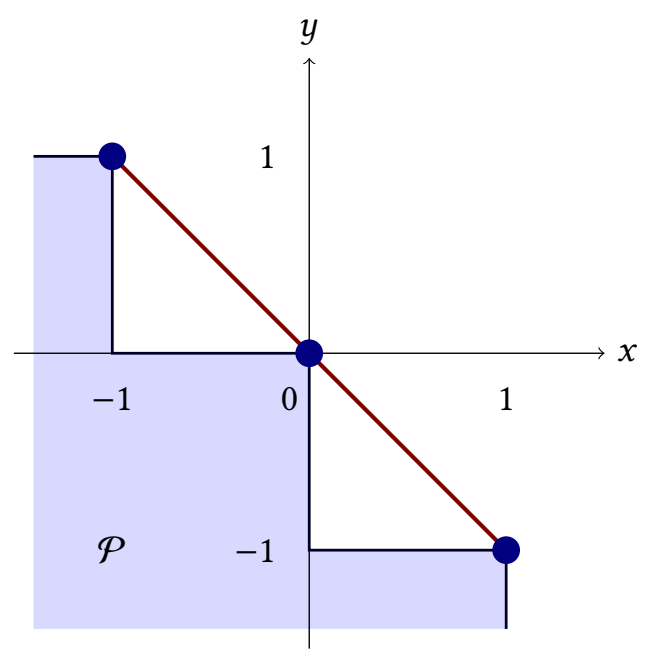

Figure 5: The production set in Example 3.7.

Now, there exists a continuum of competitive equilibria in which there is no consumption. Let $S_{X} \in \Sigma$ be a coalition of agents producing $(1,-1) ; S_{Y} \in \Sigma$ be a coalition of agents producing $(-1,1)$; and $S_{0} \in \Sigma$ be a coalition of inactive agents, generating $(0,0)$. Now, for any $0 \leqslant \lambda \leqslant \frac{1}{2}$, a coalition structure $\left(S_{X}, S_{Y}, S_{0}\right)$ satisfying $\mu\left(S_{X}\right)=\mu\left(S_{Y}\right)=\lambda$ and $\mu\left(S_{0}\right)=1-2 \lambda$ defines a social division of labour that generates a collective output of $(0,0)$. Together with an allocation $f$ with $f(a)=(0,0)$ for $a \in A$, this establishes a competitive equilibrium at equilibrium prices $p_{x}=p_{y}$.

However, none of these equilibria generates any meaningful consumption of either commodity in this economy. In particular, for $\lambda=\frac{1}{2}$, almost all agents engage in productive activities, but there is actually no consumption in this economy.

A straightforward modification of the proof of Theorem 3.6 gives conditions for existence of competitive equilibria in a continuum economy with home-based production only.

Corollary 3.8 Suppose that $\mathbb{E}=\langle(A, \Sigma, \mu), u, \mathcal{P}\rangle$ is a continuum economy such that almost every agent $a \in A$ has a strictly monotone utility function $u_{a}$ and a home-based production set $\mathcal{P}_{a}$.

Then there exists a competitive equilibrium in $\mathbb{E}$ with $f(a)>0$ for almost all agents $a \in A$.

The proof of this corollary rests on the recognition that the zero-vector acts essentially as a strict lower bound for all meaningful home-based production. This guarantees that all agents generate a positive income under positive prices, implying the boundary property of the economy's excess demand function. Under home production SIRSpec is no longer necessary to guarantee the existence of a non-trivial equilibrium. 


\section{Equilibration through the social division of labour}

Adam Smith's "Invisible Hand" metaphor extends in our model to the assignment of consumerproducers to appropriate roles in the prevailing social division of labour. There indeed emerges a strict social division of labour founded on full specialisation in production if there are Increasing Returns to Specialisation in production. This insight is formalised through the dichotomy of consumption and production under the competitive price mechanism stated in Theorem 2.6 as well as the Specialisation Theorem 2.9.

The consequences of this for the equilibration of the economy are further investigated here. In particular, we identify conditions under which the economy equilibrates through the adjustment of the social division of labour rather than through the price mechanism. In that case, rather than supply and demand determining the equilibrium price of a commodity, the supply side conditions completely determine the terms of trade, while demand is satisfied by the appropriate adjustment of the social division of labour.

This phenomenon occurs in economies in which consumer-producers have similar productive abilities when fully specialised in the production of a single output. The next example illustrates this.

Example 4.1 Consider a continuum economy $\mathbb{E}$ with two commodities $X$ and $Y$ and a population $A=[0,1]$ endowed with the standard Lebesgue measure $\lambda$. Suppose that all consumerproducers are endowed with a home-based WIRSpec production set such that $\{(4,0),(0,4)\} \subset \mathcal{P}_{a} \subset$ Conv $\{(4,0),(0,4)\}-\mathbb{R}_{+}^{2}$ for all $a \in A$. Hence, all consumer-producers have a similar ability to produce either commodity when fully specialised and these full-specialisation production plans maximise the generated income.

We denote the market price of commodity $Y$ by $p_{y} \geqslant 0$, which is stated in terms of commodity $X$, i.e., $p_{x}=1$.

Furthermore, we assume that the agents' preferences-through the standard demand problem-result in some standard (continuous and integrable) demand function $d: A \times \mathbb{R}_{+} \times \mathbb{R}_{+} \rightarrow \mathbb{R}_{++}^{2}$ such that $d\left(a, I, p_{y}\right) \gg 0$ for all $I>0$ and $p_{y}>0$, and almost all $a \in A$.

Now, let $I\left(a, p_{y}\right)$ denote the maximal income at price $p_{y} \geqslant 0$. Obviously, it is optimal to specialise in $X$ if $p_{y} \leqslant 1$ and in $Y$ if $p_{y}>1$. Hence, $I\left(a, p_{y}\right)=4$ for $p_{y} \leqslant 1$ and $I\left(a, p_{y}\right)=4 p_{y}$ for $p_{y}>1$.

We can now easily establish that in a competitive equilibrium, in order to meet the strictly positive market demand $\int d\left(\cdot, I\left(\cdot, p_{y}\right), p_{y}\right) d \lambda \gg 0$, the equilibrium price is unique and given by $p_{y}^{*}=1$, which coincides with the ratio of the output levels under full specialisation. This results in a generated income under complete specialisation of $I^{*}=I\left(a, p_{y}^{*}\right)=4$ for almost all $a \in A$.

Now, at equilibrium price $p_{y}^{*}=1$ we have $\int d\left(a, I^{*}, p_{y}^{*}\right) d \lambda(a)=\left(x^{*}, y^{*}\right) \gg(0,0)$ with $x^{*}+y^{*}=4$ and, therefore, this equilibrium is achieved through the adjustment of the social division of labour with a population fraction $v=\frac{x^{*}}{4}$ specialising in the production of $X$ and the remaining population fraction $1-v=\frac{y^{*}}{4}$ specialising in the production of $Y$.

Uniform specialisation and specialisation types. It is clear that in Example 4.1 equilibration is accomplished through adjustment of the social division of labour rather than through standard 
price equilibration. Here, we discuss the conditions under which this occurs. The next definition introduces the required notions to properly describe these situations.

Definition 4.2 Let $\mathbb{E}=\langle(A, \Sigma, \mu), u, \mathcal{P}\rangle$ be an economy such that almost all agents are endowed with a production set that satisfies WIRSpec. Denote by $Q_{a} \subset \mathbb{R}^{\ell}$ a set of $\ell$ full-specialisation production plans for agent $a \in A$ with $Q_{a} \subset \mathcal{P}_{a} \subset \operatorname{Conv} Q_{a}-\mathbb{R}_{+}^{\ell}$, as introduced in Definition 2.7. Then:

(i) Two agents $a, b \in A, a \neq b$, are specialised equivalently if both $a$ and $b$ are endowed with production sets such that their full specialisation production plans coincide: $Q_{a}=Q_{b}$.

(ii) The economy $\mathbb{E}$ is a specialisation type economy with $T \geqslant 2$ specialisation types if the agent population $A$ is partitioned in a finite number of measurable subsets $\left\{A_{1}, \ldots, A_{T}\right\}$ such that $\mu\left(A_{t}\right)>0$ and almost all agents in $A_{t}$ are specialised equivalently, $t=1, \ldots, T$, i.e., for all types $t=1, \ldots, T$ and all commodities $k=1, \ldots, \ell$, there exist full specialisation production plans $z_{t}^{k}=Q_{t}^{k} e_{k}-z_{t}^{k-} \in \mathbb{R}^{\ell}$ with for almost every $a \in A_{t}$ :

$$
Q_{t} \subset \mathcal{P}_{a} \subset \operatorname{Conv} Q_{t}-\mathbb{R}_{+}^{\ell},
$$

where $Q_{t}=\left\{z_{t}^{k}, \ldots, z_{t}^{\ell}\right\}$ is the set of $\ell$ full specialisation production plans for Type $t$ agents.

(iii) The economy $\mathbb{E}$ satisfies the uniform specialisation property if almost all agents in the population $A$ are specialised equivalently, i.e., for every commodity $k \in\{1, \ldots, \ell\}$ there exists a full specialisation production plan $z^{k}=Q^{k} e_{k}-z^{k-} \in \mathbb{R}^{\ell}$ with for almost every $a \in A$ :

$$
Q \subset \mathcal{P}_{a} \subset \operatorname{Conv} Q-\mathbb{R}_{+}^{\ell},
$$

where $Q=\left\{z^{1}, \ldots, z^{\ell}\right\}$.

Two agents that are specialised equivalently will be able to execute exactly the same production plan when they are fully specialised in the production of a single commodity. Hence, under full specialisation both agents produce the specified commodity to exactly the same standards.

A specialisation type economy is one in which there are a finite number of standards of specialisation. Here each type represents the agents that produce an output to a common standard. These types, therefore, can be interpreted as different communities in the population, which adhere to different production standards.

An economy that satisfies the uniform specialisation property is one in which full specialisation is subject to imposed external standards rather than individual abilities. Thus, under the uniform specialisation property, the economy has a single production standard. This extends the Smithian logic of uniform specialisation standards in production in comparison with the Ricardian logic that specialisation is based on purely individualistic standards (Buchanan and Yoon, 2002).

The next result specifies the consequences of uniform specialisation standards for the resulting competitive equilibria. In particular, we show that the endogenous adjustment of the social division of labour in such economies completely determine the competitive prices of all commodities. As a consequence, the production technologies induce all equilibrium prices in this economy. 
Theorem 4.3 Let $\mathbb{E}=\langle(A, \Sigma, \mu), u, \mathcal{P}\rangle$ be an economy that satisfies the properties imposed in Definition 3.1 as well as the following properties:

- Every agent $a \in A$ is endowed with a monotone utility function $u_{a}$ as well as a production set $\mathcal{P}_{a}$ that satisfies WIRSpec;

- $\mathbb{E}$ satisfies the uniform specialisation property based on a set of $\ell$ common full specialisation production plans $Q=\left\{z^{1}, \ldots, z^{\ell}\right\}$, and;

- $\mathbb{E}$ admits a competitive equilibrium $\left(f^{*}, g^{*}\right)$ such that $\int f^{*} d \mu=\int g^{*} d \mu \gg 0$.

Then the competitive equilibrium $\left(f^{*}, g^{*}\right)$ is supported by a price vector $p^{*}>0$ such that for all $k, m \in\{1, \ldots, \ell\}$ with $k \neq m: p^{*} \cdot z^{k}=p^{*} \cdot z^{m}$.

For a proof of Theorem 4.3 we refer to Appendix $\mathrm{H}$ of this paper.

Theorem 4.3 identifies the conditions under which the characteristics of the productive abilities that are present in the economy determine the equilibrium prices of all commodities in the economy. In particular, under WIRSpec, if economic agents produce the same output levels of the commodities that they are specialised in, the competitive equilibrium prices will fully reflect this socially attainable productivity level.

One of the immediate consequences of Theorem 4.3 and its proof is that all agents in this economy have exactly the same income level in equilibrium. This implies that the generated allocation of consumption bundles exhibits no envy as pointed out by Varian (1974).

Corollary 4.4 Let $\mathbb{E}=\langle(A, \Sigma, \mu), u, \mathcal{P}\rangle$ be a continuum economy satisfying the properties assumed in Theorem 4.3. Let $\left(f^{*}, g^{*}\right)$ be a competitive equilibrium with $\int f^{*} d \mu=\int g^{*} d \mu \gg 0$.

Then the equilibrium allocation $f^{*}$ of final consumption bundles is fair in the sense of Varian (1974), i.e., $f^{*}$ is envy-free as well as Pareto optimal.

The next example shows that if the assumption of WIRSpec is weakened and a specialised consumerproducer can generate multiple outputs, the main assertion of Theorem 4.3 is no longer valid.

Example 4.5 Consider a continuum economy with $A=[0,1]$ endowed with the Lebesgue measure $\lambda$ and three commodities $X, Y$ and $Z$. All consumer-producers are identical and characterised by the Cobb-Douglas utility function $u(x, y, z)=x y z^{\alpha}$, where $\alpha \geqslant 0$, and the home-based production set $\mathcal{P}=\left\{z^{1}, z^{2}, z^{3}\right\}-\mathbb{R}_{+}^{3}$ with $z^{1}=(1,0,1), z^{2}=(0,1,1)$ and $z^{3}=(0,0,2)$. We emphasise that $\mathcal{P}$ does not satisfy WIRSpec, since Good 3 is produced in all production plans in $\mathcal{P}$. Hence, Good 3 is a by-product in every production process described in $\mathcal{P}$.

In this economy two types of equilibria emerge, depending on the demand for $Z$ expressed through the value of $\alpha$ :

High demand for $Z$ with $\alpha \geqslant 2$ : In this case there emerges an equilibrium that is determined fully by supply side conditions as stated in Theorem 4.3. Equilibrium prices are determined as $p_{x}=p_{y}=p_{z}=1$ generating an income of $I=2$ for consumer-producers regardless their specialisation. Now, the fractions of consumer-producers specialising in the three full 
specialisation production plans can be determined as $\mu_{1}=\mu_{2}=\frac{2}{\alpha+2}$ and $\mu_{3}=\frac{\alpha-2}{\alpha+2}$. The consumption in equilibrium is determined as $\left(\frac{2}{\alpha+2}, \frac{2}{\alpha+2}, \frac{2 \alpha}{\alpha+2}\right)$.

Low demand for $Z$ with $\alpha<2$ : In this case there emerges an equilibrium in which consumerproducers only specialise in the two first production plans and the equilibrium prices are determined through interaction of the demand and supply sides, antithetical to the assertion of Theorem 4.3. Indeed, one can compute that in equilibrium $p_{x}=p_{y}=1$ and $p_{z}=\frac{1}{2} \alpha<1$. The generated equilibrium income now is $I_{1}=I_{2}=\frac{1}{2} \alpha+1>I_{3}=\alpha$. So, agents only specialise in one of the first two production plans. In equilibrium, the social division of labour is $\mu_{1}=\mu_{2}=\frac{1}{2}$ and $\mu_{3}=0$. The resulting consumption in equilibrium is $\left(\frac{1}{2}, \frac{1}{2}, 1\right)$.

Here, the production set does not satisfy WIRSpec due to the ability of consumer-producers to generate two outputs. This causes the exclusion of the third specialisation in equilibrium under low demand for commodity $Z$.

Non-traded commodities. We consider an example of an economy exhibiting SIRSpec that illustrates the consequences of some commodity not being traded in equilibrium, a case excluded in Theorems 4.3 and 4.8. Here, the resulting equilibrium allocation and social division of labour can be supported by multiple price vectors.

Example 4.6 Consider an economy with a continuum of economic agents $(A, \Sigma, \mu)$ and two commodities, $X$ and $Y$. All agents are endowed with the same Stone-Geary utility function and the same production set given by

$$
U(x, y)=(x+1)(y+1) \quad \text { and } \quad \mathcal{P}=\{(1,-q),(-1, Q),(0,0)\}-\mathbb{R}_{+}^{2},
$$

where $Q>q \geqslant 0$ and $q \leqslant 1$ are, respectively, the output of good $Y$ under full specialisation and the input of good $Y$ in the production of good $X$ under full specialisation. Clearly, the given production set satisfies SIRSpec.

The equilibrium prices identified in Theorem 4.3 are computed as $p_{x}^{*}=1$ and $p_{y}^{*}=\frac{2}{Q+q}$. The generated equilibrium income level of each agent is determined as $I^{*}=\frac{Q-q}{Q+q}>0$.

If $0 \leqslant q<1<Q$, demand for both goods is given by

$$
d\left(p^{*}\right)=\left(\frac{1-q}{Q+q}, \frac{Q-1}{2}\right)
$$

while, if $0 \leqslant q<Q \leqslant 1$, demand is given by

$$
d\left(p^{*}\right)=\left(\frac{Q-q}{Q+q}, 0\right) .
$$

Hence, there would no trade, consumption and production of good $Y$ for the inefficient production technologies of $Y$ described by the second case, in particular $Q \leqslant 1$.

Furthermore, for $0 \leqslant q<Q \leqslant 1$ there are multiple price vectors that support the same equilibrium allocation. For example, if $q=0$ and $Q=\frac{1}{2}$ the final consumption bundle of each agent is given by 
$f=(1,0)$ and this is supported by all equilibrium price vectors $p^{*}=\left(1, p_{y}^{*}\right)$ with $2 \leqslant p_{y}^{*} \leqslant 4$. Of these equilibrium price vectors, only $p^{*}=(1,4)$ satisfies the properties stated in Theorem 4.3.

Economies with home production. The previous analysis can be stated for economies with home-based production only. In this case, the equilibrium prices are straightforwardly determined by achievable output levels in full specialisation only. The next corollary is an immediate consequence of Proposition 2.8(b) and Theorem 4.3.

Corollary 4.7 Let $\mathbb{E}=\langle(A, \Sigma, \mu), u, \mathcal{P}\rangle$ be an economy with home-based production satisfying the properties stated in Theorem 4.3. If $\mathbb{E}$ satisfies the uniform specialisation property with $Q \subset \mathcal{P}_{a} \subset Q-\mathbb{R}_{+}^{\ell}$ for almost all $a \in A$, where $Q=\left\{Q^{1} e_{1}, \ldots, Q^{\ell} e_{\ell}\right\}$ with $Q^{k}>0$ for $k=1, \ldots, \ell$, then every competitive equilibrium $\left(f^{*}, g^{*}\right)$ with $\int f^{*} d \mu=\int g^{*} d \mu \gg 0$ can be supported by the price vector $p^{*} \gg 0$ given by

$$
p_{k}^{*}=\frac{1}{Q^{k}} \quad \text { for all } k \in\{1, \ldots, \ell\}
$$

The conclusions of Theorem 4.3 can also be extended to specialisation type economies with homebased production.

Theorem 4.8 Let $\mathbb{E}=\langle(A, \Sigma, \mu), u, \mathcal{P}\rangle$ be an economy in which every agent $a \in A$ has a monotone utility function $u_{a}$ and a home-based production set $\mathcal{P}_{a}$ that satisfies WIRspec for a set of full home-based production plans $Q_{a}=\left\{Q_{a}^{1} e_{1}, \ldots, Q_{a}^{\ell} e_{\ell}\right\}$ with $Q_{a}^{k}>0$ for $k=1, \ldots, \ell$.

If $\mathbb{E}$ is a specialisation type economy with $T \leqslant \ell-1$ types, then every competitive equilibrium $\left(f^{*}, g^{*}\right)$ with $\int f^{*} d \mu=\int g^{*} d \mu \gg 0$ can be supported by an equilibrium price vector $p^{*}>0$ such that there exists at least one type $\hat{t} \in\{1, \ldots, T\}$ and at least two commodities $k, m \in\{1, \ldots, \ell\}$ with $k \neq m$ and $p_{k}^{*} Q_{t}^{k}=p_{m}^{*} Q_{t}^{m}$.

For a proof of Theorem 4.8 we refer to Appendix I of this paper.

The requirement in Theorem 4.8 that the number of specialisation types in the economy are bounded by the number of commodities traded is binding. The next example shows that prices are determined fully by demand and supply-rather than the adaptation of the endogenous social division of labourif the number of types equals the dimension of the commodity space.

Example 4.9 Consider a continuum economy $\mathbb{E}$ with $A=[0,1]$ endowed with the Lebesgue measure $\lambda$ and $\ell \geqslant 2$ commodities. Suppose that all agents $a \in A$ are endowed with Cobb-Douglas preferences represented as

$$
u(x)=\sum_{k=1}^{\ell} \alpha_{k} \log x_{k} \quad \text { with } \alpha_{k}>0 \text { for all } k \in\{1, \ldots, \ell\} \text { and } \sum_{k=1}^{\ell} \alpha_{k}=1 .
$$

Let there be $\ell$ specialisation types $\left\{A_{t} \mid t=1, \ldots, \ell\right\}$ with $\lambda\left(A_{t}\right)=\frac{1}{\ell}$ for $t=1, \ldots, \ell$. All Type $t$ agents $a \in A_{t}$ are endowed with identical production sets given by

$$
\mathcal{P}_{t}=\operatorname{Conv}\left\{e_{1}, \ldots, e_{t-1}, Q e_{t}, e_{t+1}, \ldots, e_{\ell}\right\}-\mathbb{R}_{+}^{\ell}
$$


where

$$
Q>\frac{\max _{k} \alpha_{k}}{\min _{k} \alpha_{k}} \geqslant 1
$$

The latter condition imposes that specialisation of Type $t$ agents results in a large enough output level in this economy to satisfy all demands. In other words, none of the commodities are too scarce.

Claim: In this economy, there exists a competitive equilibrium with an equilibrium price vector given by $p_{k}^{*}=\alpha_{k}, k=1, \ldots, \ell$.

To show the claim, for the given price vector $p_{k}^{*}=\alpha_{k}, k=1, \ldots, \ell$, it is clear that the optimal decision for an agent of type $t$ is to produce only commodity $t$, i.e., $g^{*}(a)=Q e_{t}$ for almost all $a \in A_{t}$. By (19), the generated income for type $t$ agents is $I_{t}\left(p^{*}\right)=\alpha_{t} Q>\alpha_{k}$ for all $k \neq t$.

Now for almost all $a \in A_{t}$, the equilibrium demand at $p^{*}$ is given by $d_{t}\left(p^{*}\right)=\alpha_{t} Q e$ where $e=$ $(1, \ldots, 1)$. Hence, total demand from type $t$ agents is given by

$$
\int_{A_{t}} d\left(p^{*}\right) d \lambda=\frac{\alpha_{t}}{\ell} Q e
$$

and total market demand is computed as

$$
\int d\left(p^{*}\right) d \lambda=\sum_{t=1}^{\ell} \int_{A_{t}} d\left(p^{*}\right) d \lambda=\sum_{t=1}^{\ell} \frac{\alpha_{t}}{\ell} Q e=\frac{Q}{\ell} e .
$$

Furthermore, total market supply of commodity $k$ is now computed as $\lambda\left(A_{k}\right) Q=\frac{Q}{\ell}$ and, therefore, total market supply exactly covers total market demand at the selected prices. Thus, we have shown that this is indeed a competitive equilibrium in this economy.

Since $Q$ can be selected arbitrarily as long as the lower bound is honoured, we have indeed shown that the competitive equilibrium prices in this economy are not determined by the production technologies only. On the contrary, the indicated competitive equilibrium price vector is fully determined by demand-side parameters only.

Relationship to Leontief economies. The case of economies with uniform specialisation makes clear that economies with an endogenous social division of labour bridge various well-studied models in general equilibrium analysis. It is well-known from the literature on Leontief economies that for constant returns to scale production technologies the competitive equilibrium prices are fully determined by the production parameters in the input-output matrix (Intriligator, 1971, Section 9.2). Theorem 4.3 provides a characterisation of the equilibrium prices that is similar to the ones established for Leontief economies. Therefore, an economy with uniform specialisation is effectively equivalent to a Leontief economy with constant returns to scale.

In particular, under the assumption of perfect mobility of consumer-producers between specialisations and open access to the production technologies, the social division of labour is a flexible configuration that assumes the role of properly scaling production in response to market demand as is the case in Leontief economies with constant returns to scale. In this regard, the social division of 
labour in an economy with uniform specialisation emulates the Leontief production technologies under constant returns to scale, in particular their scalability.

\section{References}

Aristotle (350 BCE): The Politics: A Treatise on Government, Ernest Barker (translator). Oxford University Press, Oxford, UK, 1995 edn.

BAsile, A. (1993): "Finitely Additive Nonatomic Coalition Production Economies: Core-Walras Equivalence," International Economic Review, 34(4), 983-994.

BLIтch, C. P. (1983): “Allyn Young on Increasing Returns,” fournal of Post Keynesian Economics, 5, 359-372.

Brown, D. J. (1991): "Equilibrium Analysis with Non-convex Technologies," in Handbook of Mathematical Economics, ed. by W. Hildenbrand, and H. F. Sonnenschein, vol. IV, chap. 36. Elsevier Science Publishers, Amsterdam, The Netherlands.

Buchanan, J. M., AND Y. J. Yoon (2002): “Globalization as framed by the two logics of trade," Independent Review, 6(3), 399-405.

Cheng, W., And X. Yang (2004): "Inframarginal Analysis of Division of Labor: A Survey," fournal of Economic Behavior and Organization, 55, 137-174.

De Simone, A. (1997): "Existence of Equilibria in Finitely Additive Nonatomic Coalition Production Economies," Journal of Applied Analysis, 3(2), 249-267.

Debreu, G. (1982): "Existence of General Equilibrium," in Handbook of Mathematical Economics, ed. by K. J. Arrow, and M. D. Intriligator, vol. II, chap. 15. Elsevier Science Publishers, Amsterdam, The Netherlands.

Diamantaras, D., and R. P. Gilles (2004): “On the Microeconomics of Specialization,” fournal of Economic Behavior and Organization, 55, 223-236.

Dixit, A. K., And J. E. Stiglitz (1977): "Monopolistic Competition and Optimum Product Diversity," American Economic Review, 67, 297-308.

Gandolfo, G. (2014): International Trade Theory and Policy. Springer Verlag, second edn.

Gilles, R. P. (1996): Economic Exchange and Social Organization: The Edgeworthian Foundations of General Equilibrium Theory. Kluwer Academic Publishers, Boston, MA.

Hildenbrand, W. (1968): "The Core of an Economy with a Measure Space of Economic Agents," Review of Economic Studies, 35, 443-452.

_ (1974): Core and Equilibria of a Large Economy. Princeton University Press, Princeton, New Jersey.

Intriligator, M. D. (1971): Mathematical Optimization and Economic Theory. Prentice Hall, Englewood Cliffs, NJ.

KIM, S. (1989): "Labor Specialization and the Extent of the Market," fournal of Political Economy, 97, 692-705. 
Krugman, P. (1981): "Intra-industry Specialization and the Gains from Trade," fournal of Political Economy, 89, 959-973.

(1991): "Increasing Returns and Economic Geography," Journal of Political Economy, 99, 483-499.

(2008): "The Increasing Returns Revolution in Trade and Geography," Nobel Prize Lecture, pp. 335-348.

Locay, L. (1990): "Economic Development and the Division of Production between Households and Markets," Journal of Political Economy, 98, 965-982.

Nakahashi, W., and M. W. Feldman (2014): "Evolution of Division of Labor: Emergence of Different Activities among Group Members," fournal of Theoretical Biology, 348, 65-79.

Oddou, C. (1982): "The Core of a Coalition Production Economy," fournal of Mathematical Economics, $9,1-21$.

Plato (380 BCE): Republic. Penguin Classics, London, UK, 2007 edn.

Romer, P. M. (1986): “Increasing Returns and Long-Run Growth,” fournal of Political Economy, 94(5), $1002-1037$.

(1987): "Growth Based on Increasing Returns Due to Specialization," American Economic Review, 77, 56-62.

Smith, A. (1776): An Inquiry into the Nature and Causes of the Wealth of Nations. University of Chicago Press, Chicago, Illinois, Reprint 1976.

Sondermann, D. (1974): "Economies of Scale and Equilibria in Coalition Production Economies," fournal of Economic Theory, 8, 259-291.

Steinegger, R. (2010): An Economic Theory of the Division of Labor: From Adam Smith to Xiaokai Yang and Inframarginal Analysis. VDM Verlag Dr. Müller, Saarbrücken, Germany.

Stigler, G. J. (1951): "The Division of Labor is Limitted by the Extent of the Market" fournal of Political Economy, 59, 185-193.

Sun, G. (2012): The Division of Labor in Economics: A History, Routledge Studies in the History of Economics. Routledge, London, UK.

Sun, G., X. YANG, AND L. Zhou (2004): "General Equilibria in Large Economies with Endogenous Structure of Division of Labor," Journal of Economic Behavior and Organization, 55, 237-256.

Suzuki, T. (1995): “Nonconvex Production Economies," fournal of Economic Theory, 66, 158-177.

TodA, M. (2002): “Core and Equilibria in Non-convex Economies," Proceedings of the Institute for Mathematical Sciences Kokyu, 1264, 91-103.

Varian, H. A. (1974): "Equity, Envy, and Efficiency," fournal of Economic Theory, 9, 63-91.

WEN, M. (1998a): "An analytical framework of consumer-producers, economies of specialization and transaction costs," in Increasing Returns and Economic Analysis, ed. by K. J. Arrow, Y.-K. Ng, and X. Yang. St. Martin's Press, New York, NY.

(1998b): "The Dichotomy between Production and Consumption Decisions and Economic Efficiency," Paper presented at the Australian Economic Theory Workshop, University of Melbourne, Australia. 
YANG, X. (1988): "A Microeconomic Approach to Modeling the Division of Labor Based on Increasing Returns to Specialization," Ph.D. thesis, Princeton University, Princeton, New Jersey.

(2001): Economics: New Classical Versus Neoclassical Frameworks. Blackwell Publishing, Malden, Massachusetts.

(2003): Economic Development and the Division of Labor. Blackwell Publishing, Malden, Massachusetts.

YANG, X., AND J. Borland (1991): “A Microeconomic Mechanism for Economic Growth," fournal of Political Economy, 99, 460-482.

YANG, X., AND Y.-K. Ng (1993): Specialization and Economic Organization: A New Classical Microeconomic Framework. North-Holland, Amsterdam, Netherlands.

YAO, S. (2002): "Walrasian Equilibrium Computation, Network Formation, and the Wen Theorem," Review of Development Economics, 6, 415-427.

Young, A. A. (1928): "Increasing Returns and Economic Progress," Economic fournal, 38, 527-542. 


\section{Appendices: Proofs}

\section{A Proof of Theorem 2.6}

Assume that $\left(x^{*}, y^{*}\right)$ is a solution to the consumer-producer problem (4) for $p>0$. Then $p \cdot x^{*} \leqslant p \cdot y^{*}$ and, thus, $x^{*}$ solves the standard demand problem for income level $p \cdot y^{*}$. It remains to show that $y^{*}$ solves the income maximisation problem.

Suppose to the contrary that there is some $y^{\prime} \in \mathcal{P}$ with $p \cdot y^{\prime}>p \cdot y^{*}$. Then from $p>0$ there exists some $x^{\prime} \gg x^{*}$ with $p \cdot y^{*}<p \cdot x^{\prime} \leqslant p \cdot y^{\prime}$. Since $u$ is monotone, it follows that $u\left(x^{\prime}\right)>u\left(x^{*}\right)$, which contradicts that $x^{*}$ solves (4). Therefore, the pair $\left(x^{*}, y^{*}\right)$ solves the two-stage problem.

Suppose that $(\hat{x}, \hat{y})$ solves the two-stage income maximisation and standard demand problem for $p>0$. Assume to the contrary that $(\hat{x}, \hat{y})$ does not solve (4) for $p>0$.

Then there exist some $\left(x^{\prime}, y^{\prime}\right)$ with $y^{\prime} \in \mathcal{P}, u\left(x^{\prime}\right)>u(\hat{x})$ and $p \cdot x^{\prime} \leqslant p \cdot y^{\prime}$. Since $\hat{y}$ solves the income maximisation problem, it follows that $p \cdot y^{\prime} \leqslant p \cdot \hat{y}$. But then the fact that $u\left(x^{\prime}\right)>u(\hat{x})$ contradicts the hypothesis that $\hat{x}$ solves the demand problem for $\hat{y}$.

\section{B Proof of Proposition 2.8}

Proof of 2.8(a): WIRSpec implies that $\mathcal{P}$ is bounded from above by $\bar{Q}=\left(Q^{1}, \ldots, Q^{\ell}\right) \gg 0$. Let $q=\sum_{k=1}^{\ell} z^{k-} \geqslant 0$, then $\overline{\mathcal{P}}=\{y \in \mathcal{P} \mid \bar{Q}-q \leqslant y \leqslant \bar{Q}\}$ is a compact set such that $\mathcal{P}=\overline{\mathcal{P}}-\mathbb{R}_{+}^{\ell}$.

Proof of 2.8(b): Suppose $\mathcal{P}$ satisfies WIRSpec for $Q=\left\{z^{1}, \ldots, z^{\ell}\right\}$. Assume now that $z^{k-}>0$ for some $k \in\{1, \ldots, \ell\}$, implying that $z_{m}^{k}<0$ for some $m \neq k$. Since $\mathcal{P}$ is home-based, there is some $y \in \overline{\mathcal{P}}$ with $y \geqslant z^{k}$ and $y \geqslant 0$. This implies that $y>z^{k}$, since $y_{m} \geqslant 0>z_{m}^{k}$. This contradicts that $\mathcal{P}$ satisfies WIRSpec for $Q$. Hence, $z^{k-}=0$, showing the assertion.

Proof of 2.8(c): Suppose that $\mathcal{P} \subset \mathbb{R}^{\ell}$ satisfies SIRSpec for $Q=\left\{z^{1}, \ldots, z^{\ell}\right\} \subset \mathcal{P}$. Since all $\ell$ full specialisation production plans $z^{k}$ are independent, they are contained in a unique $(\ell-1)$ dimensional hyperplane. Hence, there exists a normal vector $h=\left(h_{1}, \ldots, h_{\ell}\right) \in \mathbb{R}^{\ell} \backslash\{0\}$ and a constant $H \in \mathbb{R}$ such that

$$
\left\{y \in \mathbb{R}^{\ell} \mid y=\sum_{k} \lambda_{k} z^{k} \text { for } \lambda=\left(\lambda_{1}, \ldots, \lambda_{\ell}\right) \text { with } \sum_{k} \lambda_{k}=1\right\}=\left\{y \in \mathbb{R}^{\ell} \mid h \cdot y=H\right\} .
$$

In particular, $h \cdot y=H$ for all $y \in \operatorname{Conv} Q$, implying that $\operatorname{Conv} Q$ is contained in the hyperplane. Since $0 \in \mathcal{P}$ implies that $\mathbb{R}_{-}^{\ell} \subset \mathcal{P}$, it follows from WIRSpec that

$$
\operatorname{Conv} Q \cap \mathbb{R}_{-}^{\ell} \subset\{0\} .
$$

If $0 \in \operatorname{Conv} Q$, then by the SIRSpec property that $\mathcal{P} \cap \operatorname{Conv} Q=Q$, it follows that $0 \in Q$. This is a contradiction. Therefore, $0 \notin \operatorname{Conv} Q$, implying that $\operatorname{Conv} Q \cap \mathbb{R}_{-}^{\ell}=\varnothing$. This implies that $h$ and $H$ can be selected such that $h \gg 0$ with $\sum_{k} h_{k}=1$ and $H>0$.

We now show that $H e \in \operatorname{Conv} Q$, implying the assertion, since $H e \gg 0$.

Since $h \cdot H e=H \sum_{k} h_{k}=H$, there exists a vector of weights $\lambda=\left(\lambda_{1}, \ldots, \lambda_{\ell}\right)$ with $\sum_{k} \lambda_{k}=1$ and $H e=\sum_{k} \lambda_{k} z^{k}$. Let $m \in\{1, \ldots, \ell\}$. Since $\sum_{k} \lambda_{k} z_{m}^{k}=H$ and $z_{m}^{m}=Q^{m}>0$, while $z_{m}^{k}=-y_{m}^{k} \leqslant 0$ for all $k \neq m$, it follows that $\lambda_{m}>0$. Thus, $\lambda \in(0,1)^{\ell}$ with $\sum_{k} \lambda_{k}=1$, implying that $H e \in \operatorname{Conv} Q$.

\section{Proof of Theorem 2.9}

Since the consumer-producer problem (4) is equivalent to the two-stage problem stated in Theorem 2.6, we can focus on the solution of the income maximisation problem only.

Let the production set $\mathcal{P} \subset \mathbb{R}^{\ell}$ satisfy WIRSpec. Let $z^{k} \in \mathcal{P}$ be a full specialisation production plan for $k \in\{1, \ldots, \ell\}$ such that $Q \subset \mathcal{P} \subset Q^{\prime}$ where $Q=\left\{z^{1}, \ldots, z^{\ell}\right\}$ and $Q^{\prime}=\operatorname{Conv} Q-\mathbb{R}_{+}^{\ell}$. 


\section{C.1 Proof of Theorem 2.9(a)}

Suppose that $y^{*} \in \mathcal{P}$ solves the income maximisation problem for the given price vector $p>0$. Then $p \cdot y^{*} \geqslant p \cdot y$ for all $y \in \mathcal{P}$. We first claim that $p \cdot y^{*} \geqslant p \cdot z$ for all $z \in Q^{\prime}$.

Suppose to the contrary that there exists some $z^{\prime} \in Q^{\prime}$ with $p \cdot z^{\prime}>p \cdot y^{*}$. From the definition of $Q^{\prime}$, we can assume without loss of generality that $z^{\prime} \in \operatorname{Conv} Q$. Thus, $z^{\prime}=\sum \lambda_{k} z^{k}$ for some weights $\sum \lambda_{k}=1$. Then for every $y \in \mathcal{P}: p \cdot z^{\prime}=\sum \lambda_{k} z^{k}>p \cdot y^{*} \geqslant p \cdot y$. In particular, $z^{k} \in \mathcal{P}$ for all $k$ and, therefore, $p \cdot z^{\prime}>p \cdot z^{k}$ for all $k$. This is a contradiction, showing that $p \cdot y^{*}=\max p \cdot Q^{\prime}$.

The claim implies that $p \cdot y^{*}=\max p \cdot Q^{\prime}$.

Given the definition of $\operatorname{Conv} Q$ as a convex hull of the corner points $z^{k}, k=1, \ldots, \ell$, and that $Q^{\prime}=\operatorname{Conv} Q-\mathbb{R}_{+}^{\ell}$, it follows that for at least one $K: p \cdot z^{K}=\max p \cdot Q^{\prime}=p \cdot y^{*}$.

\section{C.2 Proof of Theorem 2.9(b)}

Let $\mathcal{P}$ satisfy SIRSpec. Hence, $\mathcal{P} \subset \operatorname{Conv} Q-\mathbb{R}_{+}^{\ell}$ as well as $\mathcal{P} \cap(\operatorname{Conv} Q)=Q$.

With assertion 2.9(a) shown above, it follows immediately that for $p>0$ a solution $y^{*} \in \mathcal{P}$ to the income maximisation problem has to be such that $y^{*} \in \mathcal{P} \cap \operatorname{Conv} \mathcal{Q}$. Hence, $y^{*} \in \mathcal{Q}$.

\section{Proof of Proposition 2.10}

Let $Q(\phi)=\left\{\phi\left(e_{1}\right), \ldots, \phi\left(e_{\ell}\right)\right\}=\left\{\varphi_{1}(1) e_{1}, \ldots, \varphi_{\ell}(1) e_{\ell}\right\} \subset \mathcal{P}(\phi)$.

To show assertion 2.10 (a), suppose that all $\varphi_{k}, k \in\{1, \ldots, \ell\}$, are convex and that to the contrary $\mathcal{P}(\phi)$ does not exhibit WIRSpec. Then, in particular, there exists some labour time allocation $L=\left(L_{1}, \ldots, L_{\ell}\right)$ with $\sum_{k} L_{k}=1$ such that

$$
y=\phi(L)=\left(\varphi_{1}\left(L_{1}\right), \ldots, \varphi_{\ell}\left(L_{\ell}\right)\right) \notin \operatorname{Conv} Q(\phi)-\mathbb{R}_{+}^{\ell} .
$$

This implies that $y>\sum_{k} \lambda_{k} \phi\left(e_{k}\right)=\sum_{k} \lambda_{k} \varphi_{k}(1) e_{k}$ for some well-chosen non-negative weights $\lambda$ with $\sum_{k} \lambda_{k}=1$. Hence, $\varphi_{k}\left(L_{k}\right) \geqslant \lambda_{k} \varphi_{k}(1)$ for all $k$ and $\varphi_{k^{\prime}}\left(L_{k^{\prime}}\right)>\lambda_{k^{\prime}} \varphi_{k^{\prime}}(1)$ for some $k^{\prime}$.

For any $k$, by convexity of the production function $\varphi_{k}$ and the fact that $0 \leqslant L_{k} \leqslant 1$, we have that $\varphi_{k}\left(L_{k}\right)=\varphi_{k}\left(\left(1-L_{k}\right) \cdot 0+L_{k} \cdot 1\right) \leqslant L_{k} \varphi_{k}(1)$. Hence, with $\varphi_{k}(1)>0$ for $k=1, \ldots, \ell$, we conclude that $L_{k} \geqslant \lambda_{k}$ for all $k$ and that $L_{k^{\prime}}>\lambda_{k^{\prime}}$ for $k^{\prime}$. Hence, $\sum_{k} L_{k}>\sum_{k} \lambda_{k}=1$, which contradicts the assumption that $\sum_{k} L_{k}=1$. This completes the proof of the first assertion.

To show assertion $2.10(\mathrm{~b})$, let $\varphi_{k}, k \in\{1, \ldots, \ell\}$, be convex and let $\varphi_{k^{\prime}}$ be strictly convex. Then from the above, $\mathcal{P}(\phi)$ satisfies WIRSpec.

To show SIRSpec we have to prove that $\mathcal{P}(\phi) \cap(\operatorname{Conv} Q(\phi))=Q(\phi)$. Suppose to the contrary that there exists some $z \in[\mathcal{P}(\phi) \cap(\operatorname{Conv} Q(\phi))] \backslash Q(\phi)$. Then for all $k$ :

$$
0 \leqslant z_{k}=\varphi_{k}\left(L_{k}\right)-y_{k}=\lambda_{k} \varphi_{k}(1)
$$

for well-chosen $y \geqslant 0$ and $\sum_{k} \lambda_{k}=\sum_{k} L_{k}=1$. Clearly, $\lambda_{k}<1$ for all $k \in\{1, \ldots, \ell\}$, since otherwise $z \in Q$. Similarly, if $L_{k}=1$ it follows that $0 \leqslant z_{m}=\lambda_{m} \varphi_{m}(1)=-y_{m} \leqslant 0$, implying that $\lambda_{m}=0$ for any $m \neq k$. This contradicts the property that $\lambda_{k}<1$. Hence, $L_{k}<1$ for all $k$.

By convexity of $\varphi_{k}$ for all $k$ and strict convexity of $\varphi_{k^{\prime}}$, it now follows that $\varphi_{k}\left(L_{k}\right) \leqslant L_{k} \varphi_{k}(1)$ for all $k$ with strict inequality for $k^{\prime}$. Therefore, $\lambda_{k} \varphi_{k}(1) \leqslant \varphi_{k}\left(L_{k}\right) \leqslant L_{k} \varphi_{k}(1)$ for all $k$ and it is strict for $k^{\prime}$. From $\varphi_{k}(1)>0$ for all $k$, we conclude that $\lambda_{k^{\prime}}<L_{k^{\prime}}$ and $\lambda_{k} \leqslant L_{k}$ for all $k$, contradicting that $\sum_{k} \lambda_{k}=\sum_{k} L_{k}=1$.

Hence, $z \notin[\mathcal{P}(\phi) \cap(\operatorname{Conv} Q(\phi))] \backslash \mathcal{Q}(\phi)$, implying that $\mathcal{P}(\phi)$ indeed satisfies SIRSpec. 


\section{E Proof of the First Welfare Theorem 3.3}

Let $(f, g)$ be a competitive equilibrium in $\mathbb{E}$ for equilibrium price vector $p>0$. Now, suppose to the contrary that there exists some feasible allocation $\left(f^{\prime}, g^{\prime}\right)$ such that $u_{a}\left(f^{\prime}(a)\right) \geqslant u_{a}(f(a))$ for almost all $a \in A$ and there exists some non-negligible coalition $S \in \Sigma$ with $\mu(S)>0$ such that $u_{a}\left(f^{\prime}(a)\right)>u_{a}(f(a))$ for all $a \in S$.

From the assumption that all utility functions are monotone, we may apply Theorem 2.6 for all $a \in A$. Since $f(a)$ solves the standard demand problem for income level $p \cdot g(a)$ we know that by monotonicity of the preferences $p \cdot f^{\prime}(a) \geqslant p \cdot g(a)$ for almost all $a \in A$ and $p \cdot f^{\prime}(a)>p \cdot g(a)$ for $a \in S$. Hence, $p \cdot \int f^{\prime} d \mu>p \cdot \int g d \mu=p \cdot \int f d \mu$.

Furthermore, since for almost all $a \in A, g(a)$ solves $a$ 's income maximisation problem for $p$, we conclude that $p \cdot g^{\prime}(a) \leqslant p \cdot g(a)$. Therefore, $p \cdot \int g^{\prime} d \mu \leqslant p \cdot \int g d \mu$.

This implies that $p \cdot \int f^{\prime} d \mu>p \cdot \int g^{\prime} d \mu$. With $p>0$, this contradicts the feasibility of $\left(f^{\prime}, g^{\prime}\right)$, thereby showing the assertion.

\section{F Proof of the Second Welfare Theorem 3.4}

Let $\mathbb{E}=\langle(A, \Sigma, \mu), u, \mathcal{P}\rangle$ be some continuum economy and let $(f, g)$ be some Pareto optimal allocation in $\mathbb{E}$. Then for every $a \in A$ we define

$$
\Phi(a)=\left\{x \in \mathbb{R}_{+}^{\ell} \mid u_{a}(x)>u_{a}(f(a))\right\}
$$

Now $\Phi$ has measurable graph due to the measurability of all agents' preferences.

\section{F.1 Proof of Theorem 3.4(a)}

By local non-satiation of $u_{a}$, for almost all $a \in A, \Phi^{\prime}(a)=\{x \in \Phi(a) \mid 0 \leqslant x \leqslant f(a)+e\} \neq \varnothing$, where $e=(1, \ldots, 1)$. In fact, $\Phi^{\prime}$ has a measurable graph and by Aumann's selection theorem (Hildenbrand, 1974 , Theorem 1, page 54) it has a measurable selection. Since this selection is integrably bounded by $f+e$, it is integrable. Therefore, $\int \Phi^{\prime} d \mu \neq \varnothing$, implying that $\int \Phi d \mu \neq \varnothing$

By a similar argument based on Definition 3.1(iii) and (iv), $\int \mathcal{P} d \mu \neq \varnothing$. Thus, we may define

$$
\mathbf{F}=\int \Phi d \mu-\int \mathcal{P} d \mu \neq \varnothing
$$

We now claim that $\mathbf{F} \cap \mathbb{R}_{-}^{\ell}=\varnothing$. Suppose not, then there exists some $\left(f^{\prime}, g^{\prime}\right)$ with $f^{\prime}(a) \in \Phi(a)$ and $g^{\prime}(a) \in \mathcal{P}_{a}$ such that $z=\int f^{\prime} d \mu-\int g^{\prime} d \mu \leqslant 0$. Now it is easy to check that $\left(f^{\prime}, g^{\prime}\right)$ is a feasible allocation that Pareto improves upon $(f, g)$. This is a contradiction, showing the claim.

Furthermore, by Lyapunov's theorem (Hildenbrand, 1974, Theorem 3, page 62), $\mathrm{F}$ is a convex set. This allows the application of Minkowski's Separation Theorem (Hildenbrand, 1974, page 38), implying the existence of some $p>0$ such that $p \cdot z \geqslant 0$ for all $z \in \mathrm{F}$.

Define $m: A \rightarrow \mathbb{R}$ by

$$
m(a)=\inf \{p \cdot x \mid x \in \Phi(a)\} \geqslant 0
$$

By Hildenbrand (1974, Proposition 3, page 60), the function $m$ is measurable. Since preferences are locally non-satiated, for every $n \in \mathbb{N}$ there exists an integrable selection $h_{n}: A \rightarrow \mathbb{R}_{+}^{\ell}$ of $\Phi$ with $h_{n}(a) \leqslant f(a)+\frac{1}{n} e$. Now, $0 \leqslant m(a) \leqslant p \cdot h_{n}(a) \leqslant p \cdot f(a)+\frac{1}{n} p \cdot e$, implying that $m$ is integrable and that $m(a) \leqslant p \cdot f(a)$. Hence, $\int m d \mu \leqslant p \cdot \int f d \mu$.

Next define the measurable function by $I(a)=\sup p \cdot \mathcal{P}_{a} \geqslant p \cdot g(a) \geqslant 0$. Since $\mathcal{P}$ satisfies Definition 3.1 (iv), it follows that $I$ is integrably bounded and, therefore, an integrable function. 
We now introduce the transfer function $\tau: A \rightarrow \mathbb{R}$ by $\tau(a)=I(a)-m(a)$ for every $a \in A$, which is consequently integrable. It follows from the feasibility of $(f, g)$ that

$$
\int \tau d \mu=\int(I-m) d \mu \geqslant p \cdot \int(g-f) d \mu=0
$$

Now from Hildenbrand (1974, Proposition 6, page 63),

$$
0 \leqslant \inf p \cdot \mathbf{F}=\int \inf \left\{p \cdot z \mid z \in\left(\Phi(a)-\mathcal{P}_{a}\right)\right\} d \mu=\int m d \mu-\int I d \mu=-\int \tau d \mu .
$$

Hence, $\int \tau d \mu=0$.

Let $a \in A$ and $x \in \mathbb{R}_{+}^{\ell}$ be such that $u_{a}(x) \geqslant u_{a}(f(a))$. Then, by local non-satiation, for every $n \in \mathbb{N}$ there is some $x_{n} \in \Phi(a)$ with $\left\|x-x_{n}\right\|<\frac{1}{n}$. Therefore,

$$
p \cdot x_{n}+\tau(a) \geqslant m(a)+I(a)-m(a)=I(a)=\sup p \cdot \mathcal{P}_{a}
$$

Now $x_{n} \rightarrow x$ and, obviously, $p \cdot x+\tau(a) \geqslant \sup p \cdot \mathcal{P}_{a}$, showing (12).

This also implies that $p \cdot f(a)+\tau(a) \geqslant \sup p \cdot \mathcal{P}_{a} \geqslant p \cdot g(a)$ for almost all $a \in A$. This together with the properties that $\int p \cdot f(a) d \mu(a)=\int p \cdot g(a) d \mu(a)$-from feasibility of $(f, g)$-and $\int \tau d \mu=0$ leads us to (11).

This shows the assertion stated as Theorem 3.4 (a).

\section{F.2 Proof of Theorem 3.4(b)}

Let $p>0$ be the supporting price vector as constructed in the proof of Theorem 3.4 (a) above.

From SIRSpec, Proposition 2.8(c) implies that for almost all $a \in A$ there exists some $z_{a} \in$ Conv $\mathcal{P}_{a}$ with $z_{a} \gg 0$. Hence, with $p>0$ it follows that for almost all $a \in A$ :

$$
I(a)=\sup p \cdot \mathcal{P}_{a}=\sup p \cdot \operatorname{Conv} \mathcal{P}_{a} \geqslant p \cdot z_{a}>0 .
$$

From Proposition 2.8(a), $\mathcal{P}_{a}$ is delimited and, therefore, $I(a)=\max p \cdot \mathcal{P}_{a}$. Now:

Lemma F.1 For almost every $a \in A: I(a)=\max p \cdot \mathcal{P}_{a}=p \cdot g(a)>0$.

Proof. First, note that for every $a \in A$ obviously $p \cdot g(a) \leqslant \max p \cdot \mathcal{P}_{a}=I(a)$. Now suppose that $p \cdot g(a)<I(a)$ for $a \in S$ with $\mu(S)>0$. Then there exists some $g^{\prime}(a) \in \mathcal{P}_{a}$ with $p \cdot g^{\prime}(a)>p \cdot g(a)$ for $a \in A$. Hence,

$$
\Delta=\int_{S}\left[p \cdot g^{\prime}(a)-p \cdot g(a)\right] d \mu>0
$$

By strict monotonicity there exists some $f^{\prime}(a) \in \Phi(a)$ with $\left\|f^{\prime}(a)-f(a)\right\|<\frac{\Delta}{2}$. Then $z^{\prime}=\int f^{\prime} d \mu-$ $\int g^{\prime} d \mu \in \mathbf{F}$ and

$$
\begin{aligned}
p \cdot z^{\prime} & =p \cdot \int f^{\prime} d \mu-p \cdot \int g^{\prime} d \mu \leqslant p \cdot \int f d \mu+\frac{\Delta}{2}-p \cdot \int g^{\prime} d \mu< \\
& <p \cdot \int f d \mu+\Delta-p \cdot \int g^{\prime} d \mu= \\
& =p \cdot \int f d \mu+\left(p \cdot \int_{S} g^{\prime} d \mu-p \cdot \int_{S} g d \mu\right)-p \cdot \int g^{\prime} d \mu= \\
& =p \cdot \int f d \mu-p \cdot \int g d \mu=0 .
\end{aligned}
$$

This contradicts $\mathbf{F} \cap \mathbb{R}_{-}^{\ell}=\varnothing$, showing the assertion. 
From Lemma F.1 it follows that for almost every $a \in A: p \cdot f(a)+\tau(a)=p \cdot f(a)-m(a)+p \cdot g(a) \leqslant p \cdot g(a)$. Strict monotonicity of $u_{a}$ implies the following:

Lemma F.2 For almost every $a \in A: p \cdot f(a)+\tau(a)=p \cdot g(a)$.

Proof. Suppose that $p \cdot f(a)+\tau(a)<p \cdot g(a)$. Then there exists some $\varepsilon_{a}>0$ such that $x_{a}=$ $f(a)+\varepsilon_{a} e \in \mathbb{R}_{++}^{\ell}$ such that $p \cdot x_{a}+\tau(a)=p \cdot f(a)+\varepsilon_{a}+\tau(a)<p \cdot g(a)$. By strict monotonicity of $u_{a}$ it holds that $u_{a}\left(x_{a}\right)>u_{a}(f(a))$. But this contradicts the assertion of Theorem 3.4(a) shown above. This proves the lemma.

Let $T=\{a \in A \mid \tau(a) \leqslant 0\} \in \Sigma$. Since $\int \tau d \mu=0$, it follows that $\mu(T)>0$. Also, for almost every $a \in T$, from Lemma F.1, $p \cdot g(a)>0$, implying that $p \cdot f(a)>0$. Using standard arguments, e.g., Hildenbrand (1974, page 135), it follows that on $T$ a.e., $f(a)$ is a $u_{a}$-maximal bundle in the budget set $B_{a}(p)=\left\{x \in \mathbb{R}_{+}^{\ell} \mid p \cdot x+\tau(a) \leqslant p \cdot g(a)\right\}$. From the strict monotonicity of $u_{a}, p \gg 0$.

The arguments in Hildenbrand (1974, page 135) with $p \gg 0$ now imply that, for almost all $a \in A \backslash T$, $f(a)$ is a $u_{a}$-maximal bundle in the budget set $B_{a}(p)=\left\{x \in \mathbb{R}_{+}^{\ell} \mid p \cdot x+\tau(a) \leqslant p \cdot g(a)\right\}$ as well.

This shows the assertions in 3.4(b).

\section{G Proof of Existence Theorem 3.6}

The proof follows the excess demand methodology set out in Hildenbrand (1974) and Debreu (1982) for pure exchange continuum economies.

Preliminaries. Let $X$ and $Y$ be two metric spaces. By $\Psi: X \rightarrow Y$ we denote a correspondence if it assigns to every $x \in X$ some subset $\Psi(x) \subset Y$. Then:

(a) $\Psi$ is closed if for every sequence $x^{n} \rightarrow x$ in $X$ and every sequence $y^{n} \rightarrow y$ in $Y$ with $y^{n} \in \Phi\left(x^{n}\right)$ it holds that $y \in \Phi(x)$.

(b) $\Psi$ is lower hemicontinuous (lhc) if for any sequence $x^{n} \rightarrow x$ in $X$ and any $y \in \Phi(x)$ there exists a sequence $y^{n} \rightarrow y$ in $Y$ with $y^{n} \in \Phi\left(x^{n}\right)$ for large enough $n \in \mathbb{N}$.

(c) $\Psi$ is continuous if $\Phi$ is both closed as well as lhc.

Throughout we let

$$
S=\left\{p \in \mathbb{R}_{+}^{\ell} \mid \sum_{k=1}^{\ell} p_{k}=1\right\}
$$

be the $(\ell-1)$-dimensional unit simplex. $S^{o}=S \cap \mathbb{R}_{++}^{\ell}$ denotes the relative interior of $S$ and by $\partial S=\left\{p \in S \mid p_{k}=0\right.$ for some $\left.k=1, \ldots, \ell\right\}$ the boundary of $S$.

Excess demand. A correspondence $\Psi: S^{o} \rightarrow \mathbb{R}^{\ell}$ is an excess demand correspondence (Gilles, 1996, Section 6.1) if it satisfies the following three properties:

(a) Walras' Law: For every $p \in S^{o}$ it holds that $p \cdot \Psi(p)=0$.

(b) Regularity: The correspondence $\Psi$ is convex-valued, compact-valued and closed.

(c) Boundary property: For some norm $\|\cdot\|$ on $\mathbb{R}^{\ell}$, for every sequence $p^{n} \rightarrow p \in \partial S$ it holds that $\inf \left\{\|z\| \mid z \in \Psi\left(p^{n}\right)\right\} \rightarrow \infty$ as $n \rightarrow \infty$.

\section{Lemma G.1 - Excess Demand Theorem}

Any excess demand correspondence $\Psi$ has a zero point, i.e., there exists some $p^{*} \in S^{o}: 0 \in \Psi\left(p^{*}\right)$.

The excess demand theorem Lemma G.1 is stated as Theorem 8 in Debreu (1982, page 722). Slightly different proofs are provided in Debreu (1982, page 723) and Gilles (1996, page 197). 
Properties of demand and supply. Consider the continuum economy $\mathbb{E}=\langle(A, \Sigma, \mu), u, \mathcal{P}\rangle$ as asserted in Theorem 3.6. Let $a \in A$ and $p \in S$. From Definition 3.1(iii), $a$ 's production set can be rewritten as $\mathcal{P}_{a}=\overline{\mathcal{P}}_{a}-\mathbb{R}_{+}^{\ell}$, where $\overline{\mathcal{P}}: A \rightarrow \mathbb{R}^{\ell}$ is an integrably bounded compact valued correspondence that has a measurable graph. Now for the income maximalisation problem (Theorem 2.6) define

$$
\begin{aligned}
\Delta(a, p) & =\arg \max \left\{p \cdot y \mid y \in \overline{\mathcal{P}}_{a}\right\} \subset \mathcal{P}_{a} \\
I(a, p) & =\max \left\{p \cdot y \mid y \in \mathcal{P}_{a}\right\}
\end{aligned}
$$

For the standard demand problem (Theorem 2.6) we introduce

$$
\begin{aligned}
& B(a, p)=\left\{x \in \mathbb{R}_{+}^{\ell} \mid p \cdot x \leqslant I(a, p)\right\} \\
& \Gamma(a, p)=\arg \max \left\{u_{a}(x) \mid x \in B(a, p)\right\}
\end{aligned}
$$

We summarise the properties of the various concepts introduced in the following lemmas.

Lemma G.2 The following properties hold for the income function $I: A \times S \rightarrow \mathbb{R}$ and the production plan correspondence $\Delta: A \times S \rightarrow \mathbb{R}_{+}^{\ell}$.

(i) For every agent $a \in A$ and price $p \in S$ the set $\Delta(a, p) \neq \varnothing$ is compact and the generated income $I(a, p)=p \cdot \Delta(a, p)>0$ is strictly positive.

(ii) For every agent $a \in A, I(a, \cdot): S \rightarrow \mathbb{R}_{++}$is continuous and the supply correspondence $\Delta(a, \cdot): S \rightarrow R_{+}^{\ell}$ is closed.

(iii) For every price $p \in S$, the function $I(\cdot, p): A \rightarrow \mathbb{R}_{++}$is integrable and the correspondence $\Delta(\cdot, p): A \rightarrow \mathbb{R}_{+}^{\ell}$ has a measurable graph and is integrably bounded.

Proof. Let $a \in A$ and $p \in S$. Then the income maximisation problem on $\overline{\mathcal{P}}_{a}$ for $p$ has a solution by standard arguments, due to continuity of $u(a, \cdot)$ and compactness of the restricted production set $\overline{\mathcal{P}}_{a}$. The generated income $p \cdot \Delta(a, p)$ is non-negative, due to the assumption that $0 \in \overline{\mathcal{P}}_{a}$.

Also, $\mathcal{P}_{a}=\overline{\mathcal{P}}_{a}-\mathbb{R}_{+}^{\ell}$ implies that $I(a, p)=p \cdot \Delta(a, p)$.

Next, since $\mathcal{P}_{a}$ satisfies SIRSpec for some $Q_{a}$, by Proposition 2.8(c) there exists some $y \in \operatorname{Conv} Q_{a} \cap$ $\mathbb{R}_{++}^{\ell}$. This in turn implies that $I(a, p) \geqslant p \cdot y>0$. This shows assertion (i).

Berge's Maximum Theorem (Hildenbrand, 1974, Corollary, page 30) now implies that for every $a \in A$ the function $I(a, \cdot)$ is continuous on $S$ and that the correspondence $\Delta(a, \cdot)$ is closed on $S$. This, therefore, shows assertion (ii).

Furthermore, Hildenbrand (1974, Proposition 3, page 60) implies that for every $p \in S$ the function $I(\cdot, p)$ and the correspondence $\Delta(\cdot, p)$ are both measurable on $A$. The integrably boundedness of $\overline{\mathcal{P}}$ on $A$ implies that $0 \leqslant I(a, p) \leqslant p \cdot \bar{Q}(a)$ is integrably bounded as well. Thus, $I(\cdot, p)$ is integrable.

The next result completes the relevant properties for the income maximisation problem.

Lemma G.3 For every price vector $p \in S: \int \Delta(\cdot, p) d \mu \neq \varnothing$ is compact and convex.

Proof. It follows from the boundedness of the restricted production set correspondence $\overline{\mathcal{P}}$ by the integrable function $\bar{Q}$ that there exists an integrable selection in $\Delta(\cdot, p)$, implying nonemptiness of the integral of $\Delta(\cdot, p)$. Furthermore, $-\int \bar{Q} d \mu \leqslant \int \Delta(\cdot, p) d \mu \leqslant \int \bar{Q} d \mu$, which implies compactness of $\int \Delta(\cdot, p) d \mu$ by Proposition 7 in Hildenbrand (1974, page 73).

Convexity of $\int \Delta(\cdot, p) d \mu$ follows from the atomlessness of the probability space $(A, \Sigma, \mu)$ and Liapunov's Convexity Theorem, e.g., Theorem 3 in Hildenbrand (1974, page 62).

The following properties hold for the budget correspondence $B: A \times S^{o} \rightarrow \mathbb{R}_{+}^{\ell}$ and the demand correspondence $\Gamma: A \times S^{o} \rightarrow \mathbb{R}_{+}^{\ell}$. 
Lemma G.4 Let $a \in A$. Then:

(i) The budget correspondence $B(a, \cdot): S^{o} \rightarrow \mathbb{R}_{+}^{\ell}$ is continuous.

(ii) The demand correspondence $\Gamma(a, \cdot): S^{o} \rightarrow \mathbb{R}_{+}^{\ell}$ is compact-valued and closed.

Proof. We first show that $B(a, \cdot)$ is closed on $S^{o}$. Take any sequence $p^{n} \rightarrow \hat{p}$ in $S^{o}$ and let $x^{n} \in$ $B\left(a, p^{n}\right)$. Hence, $p^{n} \cdot x^{n} \leqslant I\left(a, p^{n}\right)$. So, for $n$ large enough, $p^{n} \cdot x^{n} \leqslant I(a, \hat{p})+1$.

Thus, $\left(x^{n}\right)$ is bounded and admits a convergent subsequence. Without loss of generality we may assume that there is some $\hat{x} \in \mathbb{R}_{+}^{\ell}: x^{n} \rightarrow \hat{x}$. Thus, $p^{n} \cdot x^{n} \rightarrow \hat{p} \cdot \hat{x} \leqslant I(a, \hat{p})$, i.e., $B(a, \cdot)$ is closed.

Next we show that $B(a, \cdot)$ is lhc on $S^{o}$. Take any $p^{n} \rightarrow \hat{p}$ in $S^{o}$ and any $\hat{x} \in B(a, \hat{p})$. Then we can distinguish two cases:

$\hat{p} \cdot \hat{x}<I(a, \hat{p}):$ Then for $n$ large enough we have that $p^{n} \cdot \hat{x} \leqslant I\left(a, p^{n}\right)$. Now take $x^{n}=\hat{x}$ for all $n \in \mathbb{N}$ and trivially lhc is implied at $\hat{p}$.

$\hat{p} \cdot \hat{x}=I(a, \hat{p}):$ Suppose first that $I(a, \hat{p})>0$. Then, since $I(a, \cdot)$ is continuous and $p^{n} \rightarrow \hat{p}$, we have that $I\left(a, p^{n}\right)>0$ for $n$ large enough. Now, due to strict positivity of the income $I(a, \hat{p})$ we can select some $x^{\prime}<\hat{x}$ with $\hat{p} \cdot x^{\prime}<I(a, \hat{p})=\hat{p} \cdot \hat{x}$. Now define for every $n$ the hyperplane

$$
H_{n}=\left\{z \in \mathbb{R}^{\ell} \mid p^{n} \cdot z=I\left(a, p^{n}\right)>0\right\} .
$$

Now let $z^{n} \in \mathbb{R}_{+}^{\ell}$ be the intersection point of the line through $x^{\prime}$ and $\hat{x}$ with the hyperplane $H_{n}$. Then $z^{n}$ is unique. Now define

$$
x^{n}= \begin{cases}z^{n} & \text { if } x^{\prime} \leqslant z^{n} \leqslant \hat{x} \\ \hat{x} & \text { otherwise }\end{cases}
$$

By continuity of $I(a, \cdot)$ on $S^{o}$ we have that $p^{n} \cdot x^{n} \leqslant I\left(a, p^{n}\right)$ for infinitely many $n$. Also, $I\left(a, p^{n}\right) \rightarrow I(a, \hat{p})$ and, therefore, $x^{n} \rightarrow \hat{x}$. Hence, $B(a, \cdot)$ is lhc at $\hat{p}$

Next, suppose that $I(a, \hat{p})=0$. Then, since $\hat{p} \gg 0$, it follows that $\hat{x}=0$ and, by continuity, $I\left(a, p^{n}\right) \rightarrow 0=I(a, \hat{p})$. Now define $x^{n}=I\left(a, p^{n}\right) e \geqslant 0$. Then $p^{n} \cdot x^{n}=I\left(a, p^{n}\right)$ implying that $x^{n} \in B\left(a, p^{n}\right)$. Also, $x^{n} \rightarrow 0=\hat{x}$. Therefore, $B(a, \cdot)$ is lhc at $\hat{p}$ as well.

To prove the second assertion of Lemma G.4, we remark that $B(a, \cdot)$ is compact-valued, non-empty valued and continuous. The continuity of the utility function $u_{a}$ with Berge's maximum theorem implies that $\Gamma(a, p) \neq \varnothing$ is compact for all $p \gg 0$ and that $\Gamma(a, \cdot)$ is closed on $S^{o}$.

Lemma G.5 For every strictly positive price vector $p \in S^{o}$, the demand correspondence $\Gamma(\cdot, p): A \rightarrow \mathbb{R}_{+}^{\ell}$ is integrable and $\int \Gamma(\cdot, p) d \mu \neq \varnothing$ is compact and convex.

Proof. Let $p \in S^{o}$. From Lemma G.2(iii), $0 \leqslant \int I(\cdot, p) d \mu \leqslant p \cdot \int \bar{Q} d \mu$ implying that the correspondence $B(\cdot, p): A \rightarrow \mathbb{R}_{+}^{\ell}$ has a measurable graph and has a nonempty integral.

Now from Proposition 3 in Hildenbrand (1974, page 60), the demand correspondence $\Gamma(\cdot, p)$ has a measurable graph. Also, for any $a \in A$ and any $x \in \Gamma(a, p)$ it holds that

$$
p_{k} x_{k} \leqslant p \cdot x \leqslant I(a, p) \quad \text { implying } \quad 0 \leqslant x_{k} \leqslant \frac{I(a, p)}{p_{k}}
$$

and, therefore, $\Gamma(\cdot, p)$ is bounded from below by 0 and from above by an integrable function. Hence, the assertion that the integral of $\Gamma(\cdot, p)$ is non-empty.

Furthermore, since $\Gamma(a, p)$ is compact for all $a \in A$, Proposition 7 in Hildenbrand (1974, page 73) implies that $\int \Gamma(\cdot, p) d \mu$ is compact. Finally, Liapunov's theorem and the fact that $(A, \Sigma, \mu)$ is atomless imply that $\int \Gamma(\cdot, p) d \mu$ is convex. 
Construction of an excess demand function for $\mathbb{E}$ : We define $\Psi: S^{o} \rightarrow \mathbb{R}^{\ell}$ by

$$
\Psi(p)=\int \Gamma(\cdot, p) d \mu-\int \Delta(\cdot, p) d \mu
$$

We show that the correspondence $\Psi$ is in fact an excess demand correspondence for $\mathbb{E}$.

$\Psi$ satisfies Walras' Law: For every $a \in A$ and every $p \in S^{o}: p \cdot \Delta(a, p)=I(a, p)$. Also, since the utility function $u_{a}$ is strictly monotone, it follows with standard arguments that $p \cdot \Gamma(a, p)=$ $I(a, p)$. Hence,

$$
p \cdot \Psi(p)=\int[p \cdot \Gamma(a, p)-p \cdot \Delta(a, p)] d \mu(a)=\int[I(a, p)-I(a, p)] d \mu(a)=0 .
$$

$\Psi$ satisfies regularity: Let $p \in S^{o}$. By Lemmas G.3 and G.5, $\Psi(p)$ is the difference between two non-empty, convex and compact sets, implying that $\Psi(p)$ is non-empty, convex and compact. Since for every $a \in A \Gamma(a, \cdot)$ as well as $\Delta(a, \cdot)$ are closed correspondences, it follows by Proposition 8 in Hildenbrand (1974, page 73) that $\int \Gamma(a, \cdot) d \mu(a)$ and $\int \Delta(a, \cdot) d \mu(a)$ are closed as well. This implies in turn that $\Psi$ is a closed correspondence.

$\Psi$ satisfies the boundary property: Let $\|x\|=\sum_{k=1}^{\ell}\left|x_{k}\right|$ be the regular 1-norm on $\mathbb{R}^{\ell}$.

Let $p^{n} \rightarrow \hat{p} \in \partial S$ be a sequence of prices in $S^{o}$ converging to a price vector on the boundary of the price simplex. Define for every $a \in A$

$$
f^{n}(a)=\inf \left\{\|x\| \mid x \in \Gamma\left(a, p^{n}\right)\right\} .
$$

Since $\Gamma\left(a, p^{n}\right)$ is compact by Lemma G.2(i) and $\|x\|=\sum_{k=1}^{\ell} x_{k}$ for $x \geqslant 0$, it follows that $f^{n}(a)=\min _{x \in \Gamma\left(a, p^{n}\right)} \sum_{k=1}^{\ell} x_{k}$.

By Proposition 3 in Hildenbrand (1974, page 60) and the measurability of the graph of $\Gamma\left(\cdot, p^{n}\right)$ it follows that $f^{n}$ is measurable as well. Also, from the discussion above

$$
f^{n}(a) \leqslant\left(\sum_{k=1}^{\ell} \frac{1}{p_{k}^{n}}\right) I\left(a, p^{n}\right)
$$

and, therefore, $f^{n}$ is integrably bounded and, thus, integrable. Now,

$$
\inf \left\{\|x\| \mid x \in \int \Gamma\left(a, p^{n}\right) d \mu(a)\right\}=\int \inf \left\{\|x\| \mid x \in \Gamma\left(a, p^{n}\right)\right\} d \mu(a)=\int f^{n} d \mu .
$$

By Fatou's Lemma (Hildenbrand, 1974, page 46), $\int \liminf f^{n} d \mu \leqslant \liminf \int f^{n} d \mu$ and, so,

$$
\int \liminf f^{n} d \mu \leqslant \liminf \left\{\|x\| \mid x \in \int \Gamma\left(a, p^{n}\right) d \mu(a)\right\}
$$

From Lemma G.2(i), $I(a, \hat{p})>0$ for all $a \in A$. So, by strict monotonicity of the $u_{a}, \lim _{n} f^{n}(a)=$ $\infty$. Thus, $\int \liminf f^{n} d \mu=\infty$ and, by (31), $\liminf \left\{\|x\| \mid x \in \int \Gamma\left(a, p^{n}\right) d \mu(a)\right\}=\infty$.

From Lemma G.3, $\int \Delta(\cdot, p) d \mu$ is compact for any $p \in S$. Hence, there is some $U>0$ with $\|x\| \leqslant U$ for all $x \in \int \Delta(\cdot, p) d \mu$. Therefore,

$$
\liminf \left\{\|z\| \mid z \in \Psi\left(p^{n}\right)\right\} \geqslant \liminf \left\{\|x\| \mid x \in \int \Gamma\left(a, p^{n}\right) d \mu(a)\right\}-U=\infty .
$$

This shows that $\Psi$ indeed satisfies the boundary property. 
Proof of Theorem 3.6. Above, we have shown that $\Psi$ is an excess demand correspondence. From Lemma G.1, it follows that there exists some price $p^{*} \in S^{o}$ with $0 \in \Psi\left(p^{*}\right)$, i.e., there exist integrable selections $f$ of $\Gamma\left(\cdot, p^{*}\right)$ and $g$ of $\Delta\left(\cdot, p^{*}\right)$ such that $\int f d \mu=\int g d \mu$. Since $f(a) \in \Gamma\left(a, p^{*}\right)$ and $g(a) \in \Delta\left(a, p^{*}\right)$, it follows that $(f(a), g(a))$ solves the consumer-producer problem for agent $a \in A$ at price $p^{*} \gg 0$.

Finally, from Lemma G.2(i), $I\left(a, p^{*}\right)=p^{*} \cdot f(a)>0$ for almost all $a \in A$. From $p^{*} \gg 0$ it immediately follows that $f(a)>0$ for almost all $a \in A$.

\section{H Proof of Theorem 4.3}

Let $\mathbb{E}=\langle(A, \Sigma, \mu), u, \mathcal{P}\rangle$ be some economy as postulated in Theorem 4.3. In particular, $\mathbb{E}$ admits a non-trivial competitive equilibrium $\left(f^{*}, g^{*}\right)$ with $\int f^{*} d \mu=\int g^{*} d \mu \gg 0$ such that $p^{*}>0$ is some corresponding competitive equilibrium price vector.

Since $\mathbb{E}$ satisfies the uniform specialisation property, for every $k \in\{1, \ldots, \ell\}$ there exist full specialisatuion production plans $z^{k} \in \mathbb{R}^{\ell}$ such that for almost all $a \in A: Q \subset \mathcal{P}_{a} \subset \operatorname{Conv} Q-\mathbb{R}_{+}^{\ell}$ where $Q=\left\{z^{1}, \ldots, z^{\ell}\right\}$.

Then by construction $Q$ spans an $(\ell-1)$-dimensional hyperplane in $\mathbb{R}^{\ell}$. Now, due to Theorem 2.6, for every $a \in A$ the production plan $g^{*}(a)$ solves $a$ 's income maximisation problem. Hence,

$$
p^{*} \cdot g^{*}(a)=\max p^{*} \cdot \mathcal{P}_{a}=\max p^{*} \cdot[\operatorname{Conv} Q]=\max p^{*} \cdot Q .
$$

Since $\int g^{*}(a) d \mu(a) \gg 0$ is full dimensional, that all $z \in Q$ are only positive in a single coordinate, and all agents solve exactly the same maximisation problem, namely $\max p^{*} \cdot Q$, it has to hold that every $z \in Q$ has to be a solution to that maximisation problem. Hence, $p^{*} \cdot g^{*}(a)=p^{*} \cdot z^{k}$ for all commodities $k \in\{1, \ldots, \ell\}$.

This shows the assertion stated Theorem 4.3.

\section{Proof of Theorem 4.8}

Let $\mathbb{E}=\langle(A, \Sigma, \mu), u, \mathcal{P}\rangle$ be some economy as postulated in Theorem 4.8. Assume that $\mathbb{E}$ admits a non-trivial competitive equilibrium $\left(f^{*}, g^{*}\right)$ with $\int f^{*} d \mu=\int g^{*} d \mu \gg 0$ supported by an equilibrium price $p^{*}>0$.

Assuming that $\mathbb{E}$ has home-based production only and satisfies the specialisation type property, for every type $t \in\{1, \ldots, T\}$ there exist $Q_{t}^{k}>0$ for all $k \in\{1, \ldots, \ell\}$ such that for almost all $a \in A_{t}$ :

$$
Q_{t} \subset \mathcal{P}_{a} \subset \operatorname{Conv} Q_{t}-\mathbb{R}_{+}^{\ell} \quad \text { where } Q_{t}=\left\{Q_{t}^{1} e_{1}, \ldots, Q_{t}^{\ell} e_{\ell}\right\} .
$$

Then by construction $Q_{t}$ spans an $(\ell-1)$-dimensional hyperplane in $\mathbb{R}^{\ell}$.

Since $\int g^{*} d \mu \gg 0$ is full dimensional and the total number of types is strictly less than the dimensionality of the commodity space, i.e., $T \leqslant \ell-1$, there has to be at least one type $\hat{t} \in\{1, \ldots, T\}$ and at least two commodities $k, m \in\{1, \ldots, \ell\}$ with $k \neq m$ such that

$$
\int_{A_{\hat{t}}} g_{k}^{*} d \mu>0 \text { as well as } \int_{A_{\hat{t}}} g_{m}^{*} d \mu>0
$$

Following the arguments developed in the proof of Theorem 4.3 for the identified type $\hat{t}$, we derive that for almost all $a \in A_{\hat{t}}: p^{*} \cdot g^{*}(a)=\max p^{*} \cdot Q_{\hat{t}}=p_{k}^{*} Q_{\hat{t}}^{k}=p_{m}^{*} Q_{\hat{t}}^{m}$. This completes the proof of the assertion. 